\title{
Entanglement Entropies of the quarter filled Hubbard model
}

\author{
Pasquale Calabrese \\ Dipartimento di Fisica dell'Università di Pisa and INFN, 56127 Pisa, Italy \\ Fabian H.L. Essler \\ The Rudolf Peierls Centre for Theoretical Physics, Oxford University, Oxford OX1 3NP, UK \\ Andreas M. Läuchli \\ Institut für Theoretische Physik, Universität Innsbruck, A-6020 Innsbruck, Austria

\begin{abstract}
We study Rényi and von Neumann entanglement entropies in the ground state of the one dimensional quarter-filled Hubbard model with periodic boundary conditions. We show that they exhibit an unexpected dependence on system size: for $L=4 \bmod 8$ the results are in agreement with expectations based on conformal field theory, while for $L=0 \bmod 8$ additional contributions arise. We show that these can be understood in terms of a "shell-filling" effect, and we develop a conformal field theory approach to calculate the additional contributions to the entropies. These analytic results are found to be in excellent agreement with density matrix renormalisation group computations for weak Hubbard interactions. We argue that for larger interactions the presence of a marginal irrelevant operator in the spin sector strongly affects the entropies at the finite sizes accessible numerically, and we present an effective way to take them into account.
\end{abstract}

\section{INTRODUCTION}

The Hubbard model is a central paradigm of strongly correlated electron systems. Its $1 \mathrm{D}$ version has attracted much attention for decades, because it is exactly solvable and exhibits a Mott metal to insulator transition ${ }^{1}$. The Hamiltonian for periodic boundary conditions is

$$
H_{\mathrm{Hubb}}=-t \sum_{j=1}^{L} \sum_{\sigma=\uparrow, \downarrow}\left(c_{j, \sigma}^{\dagger} c_{j+1, \sigma}+c_{j+1, \sigma}^{\dagger} c_{j, \sigma}\right)+U \sum_{j} n_{j, \uparrow} n_{j, \downarrow},
$$

where $c_{j, \sigma}^{\dagger}$ are fermionic spin- $\frac{1}{2}$ creation operators at site $j$ with spin $\sigma=\uparrow, \downarrow, n_{j, \sigma}=c_{j, \sigma}^{\dagger} c_{j, \sigma}$, and we only consider repulsive interactions $U \geq 0$. It is known from the exact solution that the ground state of (1.1) below half filling (less than one fermion per site) is metallic and the low energy physics of the model is described by a spin and charge separated Luttinger liquid ${ }^{1}$, equivalent to the semi-direct product of two conformal field theories each with central charge $c=1^{2}$ (so that the total central charge is $c=2$ ). This correspondence has proved extremely useful in characterizing the physical properties of the Hubbard model at low energies. Over the last decade or so entanglement entropies (EEs) have developed into a powerful tool for analysing many-body quantum systems, in particular in relation to quantum criticality and topological order ${ }^{3}$. In spite of this, a detailed analysis of the EEs of the $1 \mathrm{D}$ Hubbard model beyond establishing the leading behaviour ${ }^{4}$ has been missing.

In one dimensional systems described by conformal field theories (CFT) in appropriate scaling limits very general predictions for the ground state entanglement are known $[5]$. In order to be specific, let us consider the ground state $|G S\rangle$ of a finite, periodic 1D system of length $L$ and partition the latter into a finite block $A$ of length $\ell$ and its complement $\bar{A}$. The density matrix of the entire system is then $\rho=|\mathrm{GS}\rangle\langle\mathrm{GS}|$, and we will denote the reduced density matrix of block $A$ by $\rho_{A} \equiv \operatorname{Tr}_{\bar{A}}(\rho)$. Widely used measures of entanglement are the Rényi entropies

$$
S_{n}=\frac{1}{1-n} \ln \left[\operatorname{Tr} \rho_{A}^{n}\right]
$$

They encode the full information on the spectrum of $\rho_{A}{ }^{7}$, and in the limit $n \rightarrow 1$ reduce to the more widely used von Neumann entropy

$$
S_{1}=-\operatorname{Tr} \rho_{A} \ln \rho_{A} .
$$

CFT predicts that the ground state EEs $S_{n}$ are given by

$$
S_{n}=\frac{c}{6}\left(1+\frac{1}{n}\right) \ln \left(\frac{L}{\pi} \sin \frac{\pi \ell}{L}\right)+c_{n}+o(1),
$$


where $c$ is the central charge, $c_{n}$ are non-universal additive constants, and $o(1)$ denotes terms that vanish for $\ell \rightarrow \infty$. The result (1.4) is valid beyond the scaling limit, i.e. applies to lattice models underlying the CFT under consideration, as long as subsystem size $\ell$ (and also $L-\ell$ ) is large compared to the lattice spacing. The validity of 1.4 has been confirmed for a large number of quantum spin-chains and models of interacting electrons, see ${ }^{3}$ for recent reviews. We note in passing that the knowledge of the EEs has led to a deeper understanding of numerical algorithms based on matrix product states 8 and has aided the development of novel computational methods 9 .

In a recent short communication 10 , we have shown that the ground state EEs for the Hubbard model do not always follow 1.4). Ref. 10 focussed on the particular case of a quarter-filled band, i.e. one electron per two sites $N_{\uparrow}=N_{\downarrow}=L / 4$, although our findings generalize to other fillings and, in fact, to other models (indeed the same effect was probably present also in Ref. $\left.{ }^{[11}\right)$.

The main result of Ref ${ }^{10}$ is summarized in Fig. 1, where we plot the subtracted entanglement entropies $S_{1}-2 / 3 \ln L$ for a quarter-filled Hubbard model at $U=t$ for a number of different lattice lengths $L$. Interestingly, both the $L=4 \bmod 8$ and the $L=0 \bmod 8$ data exhibit scaling collapse, but to different functions. The entropy for lattice lengths $L=4 \bmod 8$ is well-described by the CFT result (1.4) with $n=1$, while for $L=0$ mod 8 there is an additional positive contribution. Interestingly, the latter can also be obtained by means of CFT 10 . The physical origin of this unusual behaviour can be traced back to a shell filling effect.

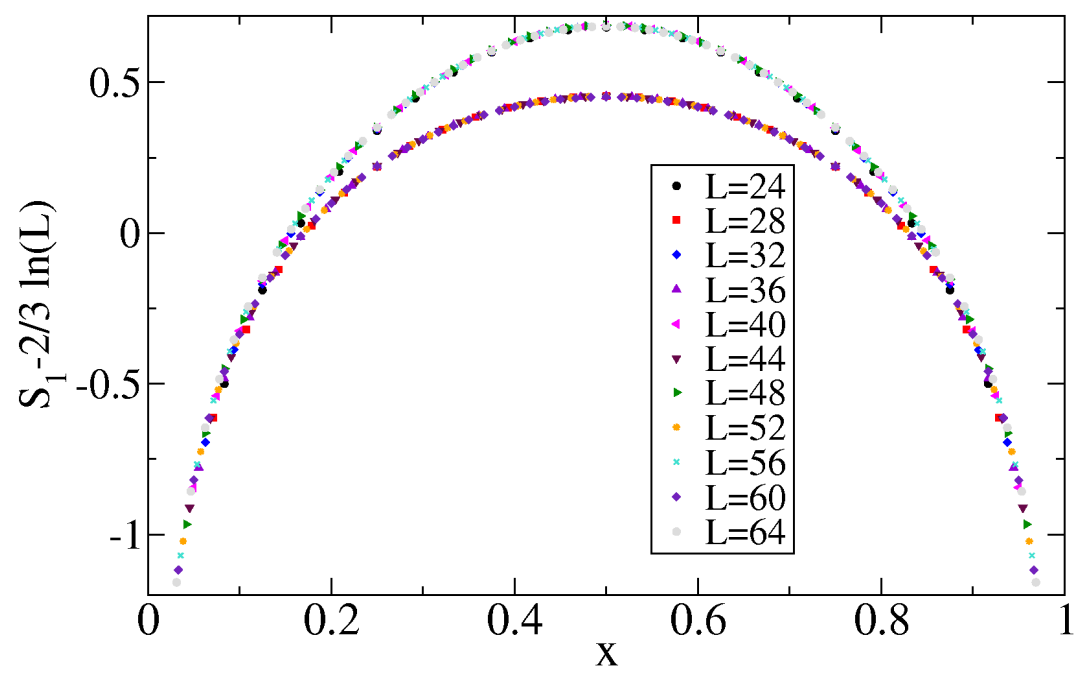

FIG. 1: DMRG data for the bipartite entanglement entropy in in the ground state of the Hubbard chain for $U=t$. We report $S_{1}-2 / 3 \ln L$ as a function of the subsystem size $x=\ell / L$ for $L=24,28,32,36,40,44,48,52,56,60,64$. The lower and upper branches corresponds to lattice lengths $L=4 \bmod 8$ and $L=0 \bmod 8$ respectively.

In this manuscript we continue our study of entanglement entropies in the 1D Hubbard model and our goal is twofold. On the one hand, we report details of the explanation and derivation of the shell filling effect in Fig. 1. which were not given previously in Ref. [10]. On the other hand, we present for the first time the results of our extensive numerical analysis of various entanglement entropies and Hubbard couplings. The manuscript is organized as follows. In Sec. II we explain the shell filling effect for the non-interacting case $U=0$ and develop a CFT description of the low-energy degrees of freedom for periodic boundary conditions and different system sizes. In Sec. III] we review elements of the exact solution of the Hubbard model, and in Sec. IV] we relate them to Luttinger liquid theory. In Sec. V] we report the CFT derivation of the entanglement entropies explaining, among the other things, the results in Fig. 1. Our analytic predictions are compared to numerical results in Sec. VI, and deviations are carefully analyzed. Finally in Sec. VIII we draw our conclusions and discuss some open issues.

\section{TIGHT-BINDING MODEL}

In order to understand the shell-filling effect in the ground state of the Hubbard Hamiltonian it is instructive to first consider the non interacting limit $(U=0)$, which is the $1 \mathrm{D}$ tight-binding model for spin- $1 / 2$ fermions

$$
H_{0}=-t \sum_{j=1}^{L} \sum_{\sigma=\uparrow, \downarrow}\left(c_{j, \sigma}^{\dagger} c_{j+1, \sigma} c_{j+1, \sigma}^{\dagger} c_{j, \sigma}\right) .
$$


$H_{0}$ is diagonalized by going to momentum space

$$
c_{\sigma}^{\dagger}(p)=\frac{1}{\sqrt{L}} \sum_{j=1}^{L} e^{-i p j} c_{j, \sigma}^{\dagger} .
$$

Imposing periodic boundary conditions leads to the quantization of the momenta

$$
p_{m}=\frac{2 \pi m}{L}, \quad-\frac{L}{2} \leq m<\frac{L}{2} .
$$

We are interested a quarter filled band and zero magnetization, i.e.

$$
N_{\uparrow}=N_{\downarrow}=L / 4 .
$$

\section{A. Ground State}

It is now straightforward to see that the precise structure of the ground state depends sensitively on whether $N_{\uparrow}=N_{\downarrow}$ are even or odd. For later convenience we introduce the notation

$$
\left|N_{\uparrow}, N_{\downarrow} ; L\right\rangle_{\mathrm{GS}}
$$

for the ground state of the Hamiltonian in the sector with $N_{\sigma}$ particles with spin $\sigma$ for a chain of length $L$.

1. Let us first consider $N_{\sigma}$ to be odd, i.e. $N_{\uparrow}=N_{\downarrow}=2 n+1$. In this case the quarter-filled ground state is unique and given by the symmetric Fermi sea

$$
|2 n+1,2 n+1 ; 8 n+4\rangle_{\mathrm{GS}}=\prod_{m=-n}^{n} c_{\uparrow}^{\dagger}\left(p_{m}\right) c_{\downarrow}^{\dagger}\left(p_{m}\right)|0\rangle,
$$

where $|0\rangle$ is the fermionic vacuum state.

2. When $N_{\sigma}=L / 4=2 n$ is even, the Fermi sea for a given spin species is necessarily asymmetric. As a consequence the ground state is not unique. The simplest way to construct the degenerate ground states is to start from the unique ground state of the Hamiltonian with $L=8 n$, but with one particle less per species, i.e. $N_{\sigma}=L / 4-1$. As $N_{\sigma}=L / 4-1$ is now odd, the ground state is the symmetric Fermi sea

$$
|2 n-1,2 n-1 ; 8 n\rangle_{\mathrm{GS}}=\prod_{m=-L / 8+1}^{L / 8-1} c_{\uparrow}^{\dagger}\left(p_{m}\right) c_{\downarrow}^{\dagger}\left(p_{m}\right)|0\rangle .
$$

The corresponding Fermi momentum is given by

$$
k_{F}=\frac{\pi}{4}=p_{L / 8}
$$

so that in the grand canonical ensemble the single-particle energy is

$$
\epsilon_{\sigma}(k)=-2 t\left[\cos (k)-\cos \left(k_{F}\right)\right] .
$$

As $\epsilon_{\sigma}\left( \pm k_{F}\right)=0$ there are four zero modes, which give rise to a total of sixteen degenerate ground states. Four of these occur at exactly quarter filling $N_{\uparrow}=N_{\downarrow}=L / 4=2 n$

$$
\begin{aligned}
|2 n, 2 n ; 8 n\rangle_{\mathrm{GS}, 1} & =c_{\uparrow}^{\dagger}\left(k_{F}\right) c_{\downarrow}^{\dagger}\left(-k_{F}\right)|2 n-1,2 n-1 ; 8 n\rangle_{\mathrm{GS}}, \\
|2 n, 2 n ; 8 n\rangle_{\mathrm{GS}, 2} & =c_{\downarrow}^{\dagger}\left(k_{F}\right) c_{\uparrow}^{\dagger}\left(-k_{F}\right)|2 n-1,2 n-1 ; 8 n\rangle_{\mathrm{GS}}, \\
|2 n, 2 n ; 8 n\rangle_{\mathrm{GS}, 3} & =c_{\uparrow}^{\dagger}\left(k_{F}\right) c_{\downarrow}^{\dagger}\left(k_{F}\right)|2 n-1,2 n-1 ; 8 n\rangle_{\mathrm{GS}}, \\
|2 n, 2 n ; 8 n\rangle_{\mathrm{GS}, 4} & =c_{\downarrow}^{\dagger}\left(-k_{F}\right) c_{\uparrow}^{\dagger}\left(-k_{F}\right)|2 n-1,2 n-1 ; 8 n\rangle_{\mathrm{GS}} .
\end{aligned}
$$

States $|2 n, 2 n ; 8 n\rangle_{\mathrm{GS}, 3}$ and $|2 n, 2 n ; 8 n\rangle_{\mathrm{GS}, 4}$ have momenta $\pm 2 k_{F}$ respectively, while $|2 n, 2 n ; 8 n\rangle_{\mathrm{GS}, 1}$ and $|2 n, 2 n ; 8 n\rangle_{\mathrm{GS}, 2}$ have momentum zero. The Hubbard interaction splits these degeneracies and selects a unique ground state in the sector with $N_{\uparrow}=N_{\downarrow}=2 n$. In the limit $U \rightarrow 0$, the Hubbard model ground state reduces to

$$
|+\rangle=\frac{1}{\sqrt{2}}\left[|2 n, 2 n ; 8 n\rangle_{\mathrm{GS}, 1}+|2 n, 2 n ; 8 n\rangle_{\mathrm{GS}, 2}\right] .
$$




\section{B. Finite-size spectrum}

In order to make contact with CFT, it is useful to have in hand expressions for the finite-size spectra of low-lying excited states. This is straightforward since we are dealing with a free fermionic theory.

$$
\text { 1. } L=8 n+4
$$

After some calculations we find

$$
E\left(\Delta N_{\sigma}, D_{\sigma}, M_{\sigma}^{ \pm}\right)=L e_{0}-\frac{\pi v}{3 L}+\frac{2 \pi v}{L} \sum_{\sigma}\left[\left(\frac{\Delta N_{\sigma}}{2}\right)^{2}+\left(D_{\sigma}\right)^{2}\right]+\frac{2 \pi v}{L} \sum_{\sigma} \sum_{n>0} n\left[M_{n, \sigma}^{+}+M_{n, \sigma}^{-}\right]
$$

where $v=\epsilon^{\prime}\left(k_{F}\right), e_{0}$ is the ground state energy density in the thermodynamic limit, $M_{n, \sigma}^{ \pm}$are integers and

$$
\Delta N_{\sigma}=N_{\sigma}-\frac{L}{4}, \quad D_{\sigma}= \begin{cases}\text { integer } & \text { if } \Delta N_{\sigma} \text { is even } \\ \text { half - odd integer } & \text { if } \Delta N_{\sigma} \text { is odd } \quad \sigma=\uparrow, \downarrow\end{cases}
$$

This means that the unique ground state is obtained by taking $N_{\sigma}=L / 4$ and $D_{\sigma}=0$, which gives the standard "conformal" result for the ground state energy

$$
E_{\mathrm{GS}}=L e_{0}-\frac{\pi v}{3 L}+o\left(L^{-1}\right)
$$

The momentum of these states is

$$
P\left(\Delta N_{\sigma}, D_{\sigma}, M_{\sigma}^{ \pm}\right)=\sum_{\sigma} \frac{2 \pi}{L}\left[\Delta N_{\sigma} D_{\sigma}+\sum_{n>0} n\left(M_{n, \sigma}^{+}-M_{n, \sigma}^{-}\right)\right]+\sum_{\sigma} 2 k_{F} D_{\sigma} .
$$

The spectra 2.12 and 2.15 are of the same form as for a compactified free boson.

$$
\text { 2. } L=8 n
$$

In this case we obtain the same expressions 2.12 and 2.15 for energy and momentum of low-lying excitations, but now the "quantum numbers" $D_{\sigma}$ take the values

$$
\Delta N_{\sigma}=N_{\sigma}-\frac{L}{4}, \quad D_{\sigma}=\left\{\begin{array}{ll}
\text { integer } & \text { if } \Delta N_{\sigma} \text { is odd } \\
\text { half }- \text { odd integer } & \text { if } \Delta N_{\sigma} \text { is even }
\end{array} \quad \sigma=\uparrow, \downarrow .\right.
$$

As discussed before, the ground state is sixteenfold degenerate in this case

$$
\begin{aligned}
& 4 \text { states: } \Delta N_{\sigma}=0, \quad D_{\sigma}= \pm \frac{1}{2}, \\
& 8 \text { states: } \Delta N_{\uparrow}= \pm 1, \Delta N_{\downarrow}=0, \quad D_{\uparrow}=0, D_{\downarrow}= \pm \frac{1}{2}, \text { and } \uparrow \leftrightarrow \downarrow, \\
& 4 \text { states: } \Delta N_{\sigma}= \pm 1, \quad D_{\sigma}=0 .
\end{aligned}
$$

\section{Correlation Functions of Local Operators for $L=8 n$}

In the case $L=8 n+4$ multi-point correlation functions of fermion operators can be easily calculated using Wick's theorem. The 2-point function is

$$
{ }_{\mathrm{GS}}\left\langle 2 n+1,2 n+1 ; 8 n+4\left|c_{j, \sigma}^{\dagger} c_{l, \tau}\right| 2 n+1,2 n+1 ; 8 n+4\right\rangle_{\mathrm{GS}}=\delta_{\sigma, \tau} \frac{\sin \left(k_{F}(j-l)\right)}{L \sin \left(\frac{\pi}{L}(j-l)\right)},
$$


where $k_{F}=\pi / 4$. The case $L=8 n$ is more complicated. Our aim is to determine correlation functions in the state 2.11. To that end it is useful to first consider correlators on a chain of length $L=8 n$ with two particles below quarter filling. In this case the symmetrically filled Fermi sea (2.7) is the unique ground-state, and we have

$$
\mathrm{GS}_{\mathrm{G}}\left\langle 2 n-1,2 n-1 ; 8 n\left|c_{j, \sigma}^{\dagger} c_{l, \tau}\right| 2 n-1,2 n-1 ; 8 n\right\rangle_{\mathrm{GS}}=\delta_{\sigma, \tau} \frac{\sin \left(\tilde{k}_{F}(j-l)\right)}{L \sin \left(\frac{\pi}{L}(j-l)\right)},
$$

where

$$
\tilde{k}_{F}=\frac{\pi}{4}-\frac{\pi}{L}
$$

As we are dealing with symmetrically filled Fermi seas, the result is the same as for ground state correlators on lattices of length $L=8 n+4$ with the replacement

$$
\tilde{k}_{F} \longrightarrow k_{F}=\frac{\pi}{4}
$$

Correlators with respect to the state $|+\rangle \sqrt{2.11}$ can now be worked out by first using the representation (2.10), and then applying Wick's theorem (in the state $|2 n-1,2 n-1 ; 8 n\rangle_{\mathrm{GS}}$ ). Let us denote the Green's function in the state $|+\rangle$ on a lattice with $L=8 n$ sites by

$$
D_{\sigma, \tau}\left(j-l, L, \tilde{k}_{F}\right)=\left\langle+\left|c_{j, \sigma}^{\dagger} c_{l, \tau}\right|+\right\rangle \text {. }
$$

Let us now consider the Green's function in the following excited state for a lattice of $L=8 n+4$ sites

$$
|\mathrm{EXC}\rangle=\frac{1}{\sqrt{2}}\left[c_{\uparrow}^{\dagger}(k) c_{\downarrow}^{\dagger}(-k)+c_{\downarrow}^{\dagger}(k) c_{\uparrow}^{\dagger}(-k)\right]|2 n+1,2 n+1 ; 8 n+4\rangle_{\mathrm{GS}},
$$

where $k$ corresponds to the lower energy empty mode, i.e.

$$
k=k_{F}+\frac{\pi}{L} .
$$

Clearly we have

$$
\left\langle\mathrm{EXC}\left|c_{j, \sigma}^{\dagger} c_{l, \tau}\right| \mathrm{EXC}\right\rangle=D_{\sigma, \tau}\left(j-l, L, k_{F}\right) .
$$

This means that rather than working with the ground-state for $L=8 n$ we can work with a particular excited state for $L=8 n+4$ and in the end of our calculations replace $\tilde{k}_{F}$ by $k_{F}$. As we are going to carry out a field theory calculation in which $k_{F}$ is an external parameter this will be straightforward to do.

\section{Field Theory Description for the noninteracting model}

Let us now think of the quarter-filled tight-binding model in the thermodynamic limit, where we have approached the latter for values of $L$ and $N_{\sigma}$ such that we are dealing with a unique ground state. A naive linearization of the fermion dispersion around the Fermi momentum gives rise to a field theory description in terms of massless Dirac fermions

$$
c_{j, \sigma} \sim \sqrt{a_{0}}\left[e^{i k_{F} x / a_{0}} R_{\sigma}(x)+e^{-i k_{F} x / a_{0}} L_{\sigma}(x)\right],
$$

where $k_{F}=\pi / 4$ and $a_{0}$ represents the lattice spacing. Here $R_{\sigma}(x)$ describes the Fourier modes of $c_{j, \sigma}$ with momenta close to $k_{F}$, while $L_{\sigma}(x)$ incorporates the modes in the vicinity of $-k_{F}$. In terms of these fields, the Hamiltonian reads

$$
H=i v \sum_{\sigma=\uparrow, \downarrow} \int d x\left[L_{\sigma}^{\dagger}(x) \partial_{x} L_{\sigma}(x)-R_{\sigma}^{\dagger}(x) \partial_{x} R_{\sigma}(x)\right],
$$

where $v=2 t a_{0} \sin \left(k_{F}\right)$ is the Fermi velocity. Periodic boundary conditions on the lattice fermions $c_{L+1}=c_{1}$ imply that for lattice lengths $L=8 n+4$

$$
R_{\sigma}\left(x+L a_{0}\right)=-R_{\sigma}(x), \quad L_{\sigma}\left(x+L a_{0}\right)=-L_{\sigma}(x) .
$$

On the other hand, for $L=8 n$ we have

$$
R_{\sigma}\left(x+L a_{0}\right)=R_{\sigma}(x), \quad L_{\sigma}\left(x+L a_{0}\right)=L_{\sigma}(x) .
$$


The Fermi fields can be bosonized ${ }^{12}$ using

$$
R_{\sigma}(x) \sim \frac{\eta_{\sigma}}{\sqrt{L a_{0}}}: e^{i \sqrt{4 \pi} \varphi_{\sigma}(x)}:, \quad L_{\sigma}(x) \sim \frac{\eta_{\sigma}}{\sqrt{L a_{0}}}: e^{-i \sqrt{4 \pi} \bar{\varphi}_{\sigma}(x)}:,
$$

where $\eta_{\sigma}$ are Klein factors. In terms of the bosonic fields the low-energy effective Hamiltonian is

$$
H=\sum_{\sigma=\uparrow, \downarrow} \frac{v}{2} \int d x\left[\left(\partial_{x} \Phi_{\sigma}\right)^{2}+\left(\partial_{x} \Theta_{\sigma}\right)^{2}\right],
$$

where we have defined canonical Bose fields $\Phi_{\sigma}$ and their dual fields $\Theta_{\sigma}$ by

$$
\Phi_{\sigma}=\varphi_{\sigma}+\bar{\varphi}_{\sigma}, \quad \Theta_{\sigma}=\varphi_{\sigma}-\bar{\varphi}_{\sigma} .
$$

Instead for working with "up" and "down" Bose fields, we can alternatively work with spin and charge bosons

$$
\begin{aligned}
& \varphi_{c}=\frac{\varphi_{\uparrow}+\varphi_{\downarrow}}{\sqrt{2}}, \quad \bar{\varphi}_{c}=\frac{\bar{\varphi}_{\uparrow}+\bar{\varphi}_{\downarrow}}{\sqrt{2}}, \quad \Phi_{c}=\varphi_{c}+\bar{\varphi}_{c}, \quad \Theta_{c}=\varphi_{c}-\bar{\varphi}_{c} \\
& \varphi_{s}=\frac{\varphi_{\uparrow}-\varphi_{\downarrow}}{\sqrt{2}}, \quad \bar{\varphi}_{s}=\frac{\bar{\varphi}_{\uparrow}-\bar{\varphi}_{\downarrow}}{\sqrt{2}}, \quad \Phi_{s}=\varphi_{s}+\bar{\varphi}_{s}, \quad \Theta_{s}=\varphi_{s}-\bar{\varphi}_{s} .
\end{aligned}
$$

In terms of these the Hamiltonian reads

$$
H=\sum_{\alpha=c, s} \frac{v}{2} \int d x\left[\left(\partial_{x} \Phi_{\alpha}\right)^{2}+\left(\partial_{x} \Theta_{\alpha}\right)^{2}\right] .
$$

The normalization of $H$ is such that, in the infinite volume limit, the two-point functions of vertex operators are normalized as

$$
\left\langle 0\left|e^{i \beta \Phi_{\alpha}(x)} e^{-i \beta^{\prime} \Phi_{\alpha}(0)}\right| 0\right\rangle \sim \delta_{\beta, \beta^{\prime}}\left[\frac{i a_{0}}{x}\right]^{\frac{\beta \beta^{\prime}}{2 \pi}} .
$$

\section{Finite-Size Spectrum an Correlations for $L=8 n+4$}

In order to impose boundary conditions and work out the finite-size energy spectrum, we recall the mode expansions of the chiral Bose fields

$$
\begin{aligned}
& \varphi_{\sigma}(x, t)=\varphi_{\sigma, 0}+\frac{x-v t}{\mathcal{L}} Q_{\sigma}+\sum_{n=1}^{\infty} \frac{1}{\sqrt{4 \pi n}}\left[e^{i \frac{2 \pi n}{\mathcal{L}}(x-v t)} a_{\sigma, R, n}+e^{-i \frac{2 \pi n}{\mathcal{L}}(x-v t)} a_{\sigma, R, n}^{\dagger}\right], \\
& \bar{\varphi}_{\sigma}(x, t)=\bar{\varphi}_{\sigma, 0}+\frac{x+v t}{\mathcal{L}} \bar{Q}_{\sigma}+\sum_{n=1}^{\infty} \frac{1}{\sqrt{4 \pi n}}\left[e^{-i \frac{2 \pi n}{\mathcal{L}}(x+v t)} a_{\sigma, L, n}+e^{i \frac{2 \pi n}{\mathcal{L}}(x+v t)} a_{\sigma, L, n}^{\dagger}\right] .
\end{aligned}
$$

Here $\mathcal{L}=L a_{0}$ in the physical length of the system, while $\varphi_{\sigma, 0}$ and $Q_{\sigma}$ are zero momentum modes satisfying canonical commutation relations

$$
\left[\varphi_{\sigma, 0}, Q_{\sigma}\right]=-\frac{i}{2}=-\left[\bar{\varphi}_{\sigma, 0}, \bar{Q}_{\sigma}\right]
$$

It is straightforward to check that the chiral Bose field fulfil the following equal time commutation relations

$$
\left[\varphi_{\sigma}(x), \varphi_{\sigma}(y)\right]=-\left[\bar{\varphi}_{\sigma}(x), \bar{\varphi}_{\sigma}(y)\right]=\frac{i}{4} \operatorname{sgn}(x-y), \quad\left[\varphi_{\sigma}(x), \bar{\varphi}_{\sigma}(y)\right]=\frac{i}{4} .
$$

We are now in a position to impose the boundary conditions 2.28 , which lead to the requirement that $e^{i \sqrt{4 \pi} \varphi_{\sigma}(\mathcal{L})}=$ $-e^{i \sqrt{4 \pi} \varphi_{\sigma}(0)}$. Substituting the mode expansions one obtains the following quantization conditions for the zero modes

$$
e^{i \sqrt{4 \pi} Q_{\sigma}}=1=e^{-i \sqrt{4 \pi} \bar{Q}_{\sigma}} .
$$


Hence the spectra of $Q_{\sigma}$ and $\bar{Q}_{\sigma}$ are

$$
q_{\sigma, m}=\sqrt{\pi} m, \quad \bar{q}_{\sigma, m^{\prime}}=\sqrt{\pi} m^{\prime}, \quad m, m^{\prime} \in \mathbb{Z} .
$$

The corresponding boundary conditions on the Bose fields $\varphi_{\sigma}(x), \bar{\varphi}_{\sigma}(x)$ are

$$
\begin{aligned}
& \varphi_{\sigma}(x+\mathcal{L})=\varphi_{\sigma}(x)+\sqrt{\pi} m_{\sigma}, \\
& \bar{\varphi}_{\sigma}(x+\mathcal{L})=\bar{\varphi}_{\sigma}(x)+\sqrt{\pi} m_{\sigma}^{\prime} .
\end{aligned}
$$

Recalling that $\Phi_{\sigma}$ is a compactified field

$$
\Phi_{\sigma}(x)=\Phi_{\sigma}(x)+\sqrt{\pi}
$$

we conclude that 2.41 in fact correspond to periodic boundary conditions on $\Phi_{\sigma}$. Substituting the mode expansions into the expression for the Hamiltonian gives

$$
H=\frac{v}{\mathcal{L}}\left[\sum_{\sigma} Q_{\sigma}^{2}+\bar{Q}_{\sigma}^{2}+\sum_{\sigma} \sum_{n>0} 2 \pi n\left(a_{\sigma, R, n}^{\dagger} a_{\sigma, R, n}+a_{\sigma, L, n}^{\dagger} a_{\sigma, L, n}\right)\right] .
$$

This results in a finite-size spectrum of the form

$$
E=\frac{2 \pi v}{\mathcal{L}}\left[\sum_{\sigma} \frac{\left(m_{\sigma}\right)^{2}+\left(m_{\sigma}^{\prime}\right)^{2}}{2}+\sum_{\sigma} \sum_{n>0} n\left[M_{n, \sigma}^{+}+M_{n, \sigma}^{-}\right]\right]
$$

Defining new quantum numbers

$$
\Delta N_{\sigma}=m_{\sigma}+m_{\sigma}^{\prime}, \quad D_{\sigma}=\frac{m_{\sigma}-m_{\sigma}^{\prime}}{2},
$$

we can rewrite the expression for the energy as

$$
E=\frac{2 \pi v}{\mathcal{L}}\left[\sum_{\sigma}\left(\frac{\Delta N_{\sigma}}{2}\right)^{2}+D_{\sigma}^{2}+\sum_{n>0} n\left[M_{n, \sigma}^{+}+M_{n, \sigma}^{-}\right]\right] .
$$

As required this reproduces the lattice result 2.12. Finally, we wish to calculate the Green's function using the field theory formalism. The ground state $|0\rangle$ is characterized by the zero mode quantum numbers $m=m^{\prime}=0$. Using the mode expansion, a straightforward calculation gives

$$
\left\langle 0\left|: e^{-i \sqrt{4 \pi} \varphi_{\sigma}(x)}:: e^{i \sqrt{4 \pi} \varphi_{\sigma}(0)}:\right| 0\right\rangle=\frac{i}{2 \sin (\pi x / \mathcal{L})}
$$

Going back to the the bosonization identities 2.30), we conclude that

$$
\begin{aligned}
\left\langle R_{\sigma}^{\dagger}(x) R_{\sigma}(0)\right\rangle & =\frac{i}{2 \mathcal{L} \sin (\pi x / \mathcal{L})}, \\
\left\langle L_{\sigma}^{\dagger}(x) L_{\sigma}(0)\right\rangle & =-\frac{i}{2 \mathcal{L} \sin (\pi x / \mathcal{L})}
\end{aligned}
$$

The asymptotics of the lattice correlators follows then to be

$$
\left\langle c_{j+\ell, \sigma}^{\dagger} c_{j, \sigma}\right\rangle \sim a_{0}\left[e^{-i k_{F} \ell}\left\langle R_{\sigma}^{\dagger}\left(\ell a_{0}\right) R_{\sigma}(0)\right\rangle+e^{i k_{F} \ell}\left\langle L_{\sigma}^{\dagger}\left(\ell a_{0}\right) L_{\sigma}(0)\right\rangle\right]=\frac{\sin \left(k_{F} \ell\right)}{L \sin (\pi \ell / L)}
$$

which, as required, agrees with the direct lattice calculation. 
3. Finite-Size Spectrum and Correlations for $L=8 n$

Following through the same steps leading to Eq. 2.40, we find that for $L=8 n$ the eigenvalues of the zero mode operators are now given by

$$
q_{\sigma, m}=\sqrt{\pi}\left(m-\frac{1}{2}\right), \quad \bar{q}_{\sigma, m}=\sqrt{\pi}\left(m-\frac{1}{2}\right), \quad m, m^{\prime} \in \mathbb{Z}
$$

These quantization conditions correspond to the following boundary conditions on the Bose fields $\varphi_{\sigma}(x), \bar{\varphi}_{\sigma}(x)$

$$
\begin{aligned}
& \varphi_{\sigma}(x+\mathcal{L})=\varphi_{\sigma}(x)+\sqrt{\pi}\left[m_{\sigma}-\frac{1}{2}\right], \\
& \bar{\varphi}_{\sigma}(x+\mathcal{L})=\bar{\varphi}_{\sigma}(x)+\sqrt{\pi}\left[m_{\sigma}^{\prime}-\frac{1}{2}\right] .
\end{aligned}
$$

The resulting energy spectrum is of the form

$$
E=\frac{2 \pi v}{L}\left[\sum_{\sigma} \frac{\left(m_{\sigma}-\frac{1}{2}\right)^{2}}{2}+\frac{\left(m_{\sigma}^{\prime}-\frac{1}{2}\right)^{2}}{2}+\sum_{\sigma} \sum_{n>0} n\left[M_{n, \sigma}^{+}+M_{n, \sigma}^{-}\right]\right] .
$$

Using the definitions 2.45 we can bring this to the same form 2.46 as for $L=8 n+4$, but now with a difference in the allowed values of $D_{\sigma}$. This again agrees with the lattice result 2.16). Our particular states of interest $|2 n, 2 n ; 8 n\rangle_{\mathrm{GS}, 1 / 2}$, cf Eq. 2.10 , correspond to quantum numbers

$$
\begin{gathered}
|2 n, 2 n ; 8 n\rangle_{\mathrm{GS}, 1}: \quad m_{\uparrow}=1=m_{\downarrow}^{\prime}, \quad m_{\downarrow}=m_{\uparrow}^{\prime}=0, \quad \Leftrightarrow \quad q_{\uparrow, 1}=\bar{q}_{\downarrow, 1}=\frac{\sqrt{\pi}}{2}, q_{\downarrow, 0}=\bar{q}_{\uparrow, 0}=-\frac{\sqrt{\pi}}{2}, \\
|2 n, 2 n ; 8 n\rangle_{\mathrm{GS}, 2}: \quad m_{\downarrow}=1=m_{\uparrow}^{\prime}, \quad m_{\downarrow}^{\prime}=m_{\uparrow}=0, \quad \Leftrightarrow \quad q_{\downarrow, 1}=\bar{q}_{\uparrow, 1}=\frac{\sqrt{\pi}}{2}, q_{\uparrow, 0}=\bar{q}_{\downarrow, 0}=-\frac{\sqrt{\pi}}{2} .
\end{gathered}
$$

The calculation of correlators in any of the sixteen ground states for $L=8 n$ proceeds in the same way as for $L=8 n+4$, cf Sec. IID 2, The only difference arises from factors like

$$
\left\langle q_{\sigma, m}, \bar{q}_{\sigma, m^{\prime}}\left|e^{-i \sqrt{4 \pi} Q_{\sigma} x / \mathcal{L}}\right| q_{\sigma, m}, \bar{q}_{\sigma, m^{\prime}}\right\rangle .
$$

If we consider the state with $m=m^{\prime}=0$ then this produces an additional factor $e^{i \pi x / \mathcal{L}}$ for the two point function of right moving fermions and a factor $e^{-i \pi x / \mathcal{L}}$ for the left movers. We then obtain the result

$$
{ }_{\mathrm{GS}}\left\langle 2 n-1,2 n-1 ; 8 n\left|c_{j+\ell, \sigma}^{\dagger} c_{j, \sigma}\right| 2 n-1,2 n-1 ; 8 n\right\rangle_{\mathrm{GS}} \sim \frac{\sin \left(\tilde{k}_{F} \ell\right)}{L \sin (\pi \ell / L)},
$$

where $\tilde{k}_{F}$ is given by 2.20 . Hence we again reproduce the correct lattice result.

\section{Excited State for $L=8 n+4$}

The excited state $|\mathrm{EXC}\rangle$ in Eq. 2.23 corresponds in the field theory limit to a linear combination of states with

$$
\Delta N_{\uparrow}=\Delta N_{\downarrow}=1, \quad D_{\uparrow}=-D_{\downarrow}=\frac{1}{2}, \quad \Leftrightarrow \quad m_{\uparrow}=1, m_{\uparrow}^{\prime}=0, \quad m_{\downarrow}=0, m_{\downarrow}^{\prime}=1,
$$

and

$$
\Delta N_{\uparrow}=\Delta N_{\downarrow}=1, \quad D_{\uparrow}=-D_{\downarrow}=-\frac{1}{2}, \quad \Leftrightarrow \quad m_{\uparrow}=0, m_{\uparrow}^{\prime}=1, \quad m_{\downarrow}=1, m_{\downarrow}^{\prime}=0 .
$$

In order to obtain a representation on the bosonic Fock space, it is convenient to employ mode the expansions in Euclidean space

$$
\begin{aligned}
& \varphi_{\sigma}(z)=\varphi_{\sigma, 0}+\frac{i}{2 \pi} \ln (z) Q_{\sigma}+\sum_{n=1}^{\infty} \frac{1}{\sqrt{4 \pi n}}\left[z^{-n} a_{\sigma, R, n}+z^{n} a_{\sigma, R, n}^{\dagger}\right] \\
& \bar{\varphi}_{\sigma}(\bar{z})=\bar{\varphi}_{\sigma, 0}-\frac{i}{2 \pi} \ln (\bar{z}) \bar{Q}_{\sigma}+\sum_{n=1}^{\infty} \frac{1}{\sqrt{4 \pi n}}\left[\bar{z}^{-n} a_{\sigma, L, n}+\bar{z}^{n} a_{\sigma, L, n}^{\dagger}\right]
\end{aligned}
$$


where we have defined complex coordinates

$$
z=e^{\frac{2 \pi}{\mathcal{L}}(v \tau-i x)}, \quad \bar{z}=e^{\frac{2 \pi}{\mathcal{L}}(v \tau+i x)} .
$$

Let us now consider the particular class of states

$$
|\alpha, \bar{\alpha}, \sigma\rangle \equiv \lim _{z, \bar{z} \rightarrow 0} e^{i \alpha \varphi_{\sigma}(z)+i \bar{\alpha} \bar{\varphi}_{\sigma}(\bar{z})}|0\rangle .
$$

In the limit $z, \bar{z} \rightarrow 0$ the oscillator modes drop out as only the annihilation operators survive. Thus

$$
\begin{aligned}
Q_{\sigma}|\alpha, \bar{\alpha}, \sigma\rangle & =-\frac{\alpha}{2}|\alpha, \bar{\alpha}, \sigma\rangle, \\
\bar{Q}_{\sigma}|\alpha, \bar{\alpha}, \sigma\rangle & =\frac{\bar{\alpha}}{2}|\alpha, \bar{\alpha}, \sigma\rangle, \\
a_{\sigma, R / L, n}|\alpha, \bar{\alpha}, \sigma\rangle & =0 .
\end{aligned}
$$

Using these results, we conclude that in the bosonized theory the excited state $|\mathrm{EXC}\rangle$ in Eq. 2.23) corresponds to

$$
a e^{-i \sqrt{4 \pi} \varphi_{\uparrow}(0)+i \sqrt{4 \pi} \bar{\varphi}_{\downarrow}(0)}|0\rangle+b e^{-i \sqrt{4 \pi} \varphi_{\downarrow}(0)+i \sqrt{4 \pi} \bar{\varphi}_{\uparrow}(0)}|0\rangle
$$

where $a$ and $b$ are complex numbers. As our state should be symmetric under interchange of up and down spins we must have that $a=b$. In terms of spin and charge bosons we conclude that

$$
\begin{gathered}
|\mathrm{EXC}\rangle \sim e^{-i \sqrt{2 \pi} \Theta_{c}(0,0)} \cos \left(\sqrt{2 \pi} \Phi_{s}(0,0)\right)|0\rangle . \\
\text { 5. Ground State }|+\rangle \text { for } L=8 n
\end{gathered}
$$

Finally, we are in a position to express our particular state of interest $|+\rangle \sqrt{2.11}$ in the bosonized theory. Employing Eqns (2.60) and (2.61), we conclude that the states (2.53) correspond to

$$
\begin{aligned}
|2 n, 2 n ; 8 n\rangle_{\mathrm{GS}, 1} \sim e^{-i \sqrt{\pi}\left(\varphi_{\uparrow}(0,0)-\varphi_{\downarrow}(0,0)-\bar{\varphi}_{\downarrow}(0,0)+\bar{\varphi}_{\uparrow}(0,0)\right)}|0\rangle \sim e^{-i \sqrt{2 \pi} \Phi_{s}(0,0)}|0\rangle, \\
|2 n, 2 n ; 8 n\rangle_{\mathrm{GS}, 2} \sim e^{-i \sqrt{\pi}\left(\varphi_{\downarrow}(0,0)-\varphi_{\uparrow}(0,0)-\bar{\varphi}_{\uparrow}(0,0)+\bar{\varphi}_{\downarrow}(0,0)\right)}|0\rangle \sim e^{i \sqrt{2 \pi} \Phi_{s}(0,0)}|0\rangle
\end{aligned}
$$

where $|0\rangle$ is the boson vacuum (which has zero mode eigenvalues zero). We note that for $L=8 n$ the vacuum state $|0\rangle$ is not an allowed state of the compact boson theory, and expressions 2.64 must be understood on an extended bosonic Fock space. We conclude that the state 2.11 has the bosonic representation

$$
|+\rangle \sim \cos \left(\sqrt{2 \pi} \Phi_{s}(0,0)\right)|0\rangle .
$$

\section{HUBBARD MODEL}

We now turn to the Hubbard model

$$
H(U)=-t \sum_{j, \sigma}\left(c_{j, \sigma}^{\dagger} c_{j+1, \sigma}+c_{j+1, \sigma}^{\dagger} c_{j, \sigma}\right)+U \sum_{j} n_{j, \uparrow} n_{j, \downarrow}-\mu \sum_{j} n_{j}
$$

where $n_{j, \sigma}=c_{j, \sigma}^{\dagger} c_{j, \sigma}$ and $n_{j}=n_{j, \uparrow}+n_{j, \downarrow}$ and we impose periodic boundary conditions. The Hubbard model is solvable by the Bethe Ansatz, and its particular eigenstates relevant to our discussion are parametrized in terms of the solutions $\left\{\Lambda_{\alpha}, k_{j}\right\}$ of the following set of coupled Bethe Ansatz Equations equations $1 \mid 13$

$$
\begin{aligned}
& k_{j} L=2 \pi I_{j}-\sum_{\alpha=1}^{M} \theta\left(\frac{\sin k_{j}-\Lambda_{\alpha}}{u}\right), \quad j=1, \ldots, N \\
& \sum_{j=1}^{N} \theta\left(\frac{\Lambda_{\alpha}-\sin k_{j}}{u}\right)=2 \pi J_{\alpha}+\sum_{\beta=1}^{M} \theta\left(\frac{\Lambda_{\alpha}-\Lambda_{\beta}}{2 u}\right), \alpha=1, \ldots, M .
\end{aligned}
$$


Here $u=U / 4 t$ is a dimensionless interaction strength, the length of the lattice $L$ is taken to be even, $\theta(x)=2 \arctan (x)$ and $N=N_{\uparrow}+N_{\downarrow}$. The quantum numbers $I_{j}, J_{\alpha}$ are integer or half-odd integer numbers that arise due to the multivaluedness of the logarithm. They are subject to the "selection rules"

$$
\begin{gathered}
I_{j} \text { is } \begin{cases}\text { integer } & \text { if } M \text { is even } \\
\text { half -odd integer } & \text { if } M \text { is odd, }\end{cases} \\
J_{\alpha} \text { is } \begin{cases}\text { integer } & \text { if } N-M \text { is odd } \\
\text { half }- \text { odd integer } & \text { if } N-M \text { is even, }\end{cases}
\end{gathered}
$$

and have ranges

$$
-\frac{L}{2}<I_{j} \leq \frac{L}{2}, \quad\left|J_{\alpha}\right| \leq \frac{1}{2}(N-M-1)
$$

The energy and momentum of such Bethe ansatz states are

$$
\begin{aligned}
& E=-\sum_{j=1}^{N}\left[2 t \cos \left(k_{j}\right)+\mu\right] \\
& P=\sum_{j=1}^{N} k_{j} \equiv \frac{2 \pi}{L}\left[\sum_{j=1}^{N} I_{j}+\sum_{\alpha=1}^{M} J_{\alpha}\right] .
\end{aligned}
$$

Following Refs! 14 , we define regular Bethe Ansatz states as eigenstates of $H(U)$ arising from solutions of 3.2 with $2 M \leq N$, where all $k_{j}$ and $\Lambda_{\alpha}$ are finite. We denote these states by

$$
\left|\left\{I_{j}\right\} ;\left\{J_{\alpha}\right\}\right\rangle_{\text {reg }}
$$

It was shown in Refs. ${ }^{14}$ that regular Bethe Ansatz states are highest weight states with respect to the $\mathrm{SO}(4)$ symmetry of the Hubbard model, i.e.

$$
\begin{array}{r}
\eta\left|\left\{I_{j}\right\} ;\left\{J_{\alpha}\right\}\right\rangle_{\mathrm{reg}}=0 \\
S^{+}\left|\left\{I_{j}\right\} ;\left\{J_{\alpha}\right\}\right\rangle_{\mathrm{reg}}=0
\end{array}
$$

where

$$
S^{+}=\sum_{j=1}^{L} c_{j, \uparrow}^{\dagger} c_{j, \downarrow}, \quad \eta=\sum_{j=1}^{L}(-1)^{j} c_{j, \uparrow} c_{j, \downarrow}
$$

A complete set of eigenstates is obtained by acting with lowering operators on the lowest weight states 14

$$
\left(S^{-}\right)^{m}\left(\eta^{\dagger}\right)^{n}\left|\left\{I_{j}\right\} ;\left\{J_{\alpha}\right\}\right\rangle_{\mathrm{reg}} .
$$

\section{A. Quarter-Filled Ground State for $L=4 \bmod 8$.}

For $L=8 n+4$ we have $N=L / 2=4 n+2$ and $M=2 n+1$. Hence the $I_{j}$ 's are half-odd integers and the $J_{\alpha}$ 's are integers. The ground state is characterised by the quantum numbers

$$
\begin{aligned}
I_{j} & =-2 n-\frac{3}{2}+j, j=1, \ldots, 4 n+2, \\
J_{\alpha} & =-n-1+\alpha, \alpha=1, \ldots, 2 n+1 .
\end{aligned}
$$

Importantly, the distributions of $I_{j}$ and $J_{\alpha}$ are symmetric around zero. The ground state is a regular Bethe Ansatz state and has total $\operatorname{spin} S=0$, i.e.

$$
|\mathrm{GS}\rangle=\left|\left\{I_{1}, \ldots, I_{\frac{L}{2}}\right\} ;\left\{J_{1}, \ldots, J_{\frac{L}{4}}\right\}\right\rangle_{\mathrm{reg}}
$$


The excitation spectrum relative to the ground state has been derived in Ref.2 and is given by

$$
\Delta E=\frac{2 \pi v_{c}}{L}\left[\frac{\left(\Delta N_{c}\right)^{2}}{4 \xi^{2}}+\xi^{2}\left(D_{c}+\frac{D_{s}}{2}\right)^{2}+N_{c}^{+}+N_{c}^{-}\right]+\frac{2 \pi v_{s}}{L}\left[\frac{\left(\Delta N_{s}-\frac{\Delta N_{c}}{2}\right)^{2}}{2}+\frac{D_{s}^{2}}{2}+N_{s}^{+}+N_{s}^{-}\right]+o\left(L^{-1}\right)
$$

where $\Delta N_{\alpha}, 2 D_{\alpha}$ and $N_{\alpha}^{ \pm}$are integer "quantum numbers" subject to the selection rules

$$
N_{\alpha}^{ \pm} \in \mathbb{N}_{0}, \quad \Delta N_{\alpha} \in \mathbb{Z}, \quad D_{c}=\frac{\Delta N_{c}+\Delta N_{s}}{2} \bmod 1, D_{s}=\frac{\Delta N_{c}}{2} \bmod 1 .
$$

Here $\xi=\xi(Q)$ is obtained from the solution of the integral equation

$$
\xi(k)=1+\int_{-Q}^{Q} d k^{\prime} \cos \left(k^{\prime}\right) R\left(\sin (k)-\sin \left(k^{\prime}\right)\right) \xi\left(k^{\prime}\right),
$$

where

$$
R(x)=\int_{-\infty}^{\infty} \frac{d \omega}{2 \pi} \frac{e^{i \omega x}}{1+\exp (2 u|\omega|)}
$$

B. Quarter-Filled Ground State for $L=0 \bmod 8$.

For $L=8 n$ we have $N=L / 2=4 n$ and $M=2 n$. Hence the $I_{j}$ 's are integers and the $J_{\alpha}$ 's are half-odd integers. One may naively expect the ground state to be obtained by choosing either

$$
\begin{aligned}
& I_{j}^{(1)}=-2 n+j, j=1, \ldots, 4 n \\
& J_{\alpha}^{(1)}=-n-\frac{1}{2}+\alpha, \alpha=1, \ldots, 2 n
\end{aligned}
$$

or

$$
\begin{aligned}
& I_{j}^{(2)}=-2 n-1+j, j=1, \ldots, 4 n, \\
& J_{\alpha}^{(2)}=-n-\frac{1}{2}+\alpha, \alpha=1, \ldots, 2 n .
\end{aligned}
$$

Comparison of their energies to numerical results for the ground state energy shows that this is not the case. The ground state is in fact obtained as follows. We start by considering regular Bethe Ansatz states with one fewer down spin, i.e. $N=L / 2=4 n$ and $M=2 n-1$. Now the $I_{j}$ 's are half-odd integers and the $J_{\alpha}$ 's are integers. The lowest energy regular Bethe Ansatz state corresponds to the choice

$$
\begin{aligned}
& I_{j}^{(0)}=-2 n-\frac{1}{2}+j, j=1, \ldots, 4 n, \\
& J_{\alpha}^{(0)}=-n+\alpha, \alpha=1, \ldots, 2 n-1 .
\end{aligned}
$$

We denote its energy by $E\left(\left\{I_{j}^{(0)}\right\} ;\left\{J_{\alpha}^{(0)}\right\}\right)$. The corresponding state is an eigenstate of $S^{z}$ with eigenvalue 1

$$
S^{z}\left|\left\{I_{j}^{(0)}\right\} ;\left\{J_{\alpha}^{(0)}\right\}\right\rangle_{\mathrm{reg}}=\left|\left\{I_{j}^{(0)}\right\} ;\left\{J_{\alpha}^{(0)}\right\}\right\rangle_{\mathrm{reg}}
$$

As we are dealing with a regular Bethe Ansatz state, the theorem of Ref 14 applies and we may conclude that we are dealing with the highest weight state of a spin-SU(2) triplet. The ground state of the quarter-filled Hubbard model with $L=8 n$ is then obtained as the $S^{z}=0$ state of this spin triplet

$$
|\mathrm{GS}\rangle=S^{-}\left|\left\{I_{j}^{(0)}\right\} ;\left\{J_{\alpha}^{(0)}\right\}\right\rangle_{\mathrm{reg}} .
$$




\section{LUTTINGER LIQUID DESCRIPTION OF THE HUBBARD CHAIN}

The bosonization of the Hubbard model proceeds by first bosonizing the theory at $U=0$, and then taking the interactions into account $\frac{12}{2}$. The result of this analysis is a spin-charge separated Luttinger liquid Hamiltonian of the form

$$
\mathcal{H}=\sum_{\alpha=c, s} \frac{v_{\alpha}}{2} \int d x\left[\left(\partial_{x} \Phi_{\alpha}\right)^{2}+\left(\partial_{x} \Theta_{\alpha}\right)^{2}\right]
$$

where the spin and charge fields are now given by

$$
\begin{array}{ll}
\Phi_{c}=\frac{\Phi_{\uparrow}+\Phi_{\downarrow}}{\sqrt{2 K}}, & \Theta_{c}=\sqrt{\frac{K}{2}}\left(\Theta_{\uparrow}+\Theta_{\downarrow}\right), \\
\Phi_{s}=\frac{\Phi_{\uparrow}-\Phi_{\downarrow}}{\sqrt{2}}, & \Theta_{s}=\frac{\Theta_{\uparrow}-\Theta_{\downarrow}}{\sqrt{2}} .
\end{array}
$$

The normalization of $H$ is such that Eq. 2.35 is satisfied by the fields $\Phi_{c, s}$. The Luttinger parameter $K$ and the velocities $v_{c, s}$ depend on the interaction strength $U$ (actually on $U / t$ ) and they can be calculated by solving appropriate integral equations (see e.g! ${ }^{1}$ ). In fact $K$ is related to the solution $\xi(k)$ of the integral equation (3.15) by

$$
K=\frac{\xi(Q)^{2}}{2}
$$

The mode expansions for the spin and charge bosons are

$$
\begin{aligned}
& \varphi_{\mathrm{a}}(x, t)=\varphi_{\mathrm{a}, 0}+\frac{x-v t}{\mathcal{L}} Q_{\mathrm{a}}+\sum_{n=1}^{\infty} \frac{1}{\sqrt{4 \pi n}}\left[e^{i \frac{2 \pi n}{\mathcal{L}}(x-v t)} a_{\mathrm{a}, R, n}+e^{-i \frac{2 \pi n}{\mathcal{L}}(x-v t)} a_{\mathrm{a}, R, n}^{\dagger}\right], \\
& \bar{\varphi}_{\mathrm{a}}(x, t)=\bar{\varphi}_{\mathrm{a}, 0}+\frac{x+v t}{\mathcal{L}} \bar{Q}_{\mathrm{a}}+\sum_{n=1}^{\infty} \frac{1}{\sqrt{4 \pi n}}\left[e^{-i \frac{2 \pi n}{\mathcal{L}}(x+v t)} a_{\mathrm{a}, L, n}+e^{i \frac{2 \pi n}{\mathcal{L}}(x+v t)} a_{\mathrm{a}, L, n}^{\dagger}\right]
\end{aligned}
$$

where $\mathrm{a}=c, s$ and the zero momentum mode operators have commutation relations

$$
\left[\varphi_{\mathrm{a}, 0}, Q_{\mathrm{a}}\right]=-\frac{i}{2}=-\left[\bar{\varphi}_{\mathrm{a}, 0}, \bar{Q}_{\mathrm{a}}\right]
$$

The spin and charge zero mode operators are related to the up and down zero mode operators by the canonical transformation

$$
\begin{aligned}
Q_{c} & =\frac{K+1}{\sqrt{4 K}} \frac{Q_{\uparrow}+Q_{\downarrow}}{\sqrt{2}}+\frac{1-K}{\sqrt{4 K}} \frac{\bar{Q}_{\uparrow}+\bar{Q}_{\downarrow}}{\sqrt{2}}, \\
\bar{Q}_{c} & =\frac{1-K}{\sqrt{4 K}} \frac{Q_{\uparrow}+Q_{\downarrow}}{\sqrt{2}}+\frac{K+1}{\sqrt{4 K}} \frac{\bar{Q}_{\uparrow}+\bar{Q}_{\downarrow}}{\sqrt{2}}, \\
Q_{s} & =\frac{Q_{\uparrow}-Q_{\downarrow}}{\sqrt{2}}, \\
\bar{Q}_{s} & =\frac{\bar{Q}_{\uparrow}-\bar{Q}_{\downarrow}}{\sqrt{2}} .
\end{aligned}
$$

The mode expansion of the Hamiltonian is

$$
\mathcal{H}=\sum_{\mathrm{a}=c, s} \frac{v_{\mathrm{a}}}{\mathcal{L}}\left[Q_{\mathrm{a}}^{2}+\bar{Q}_{\mathrm{a}}^{2}+\sum_{n>0} 2 \pi n\left(a_{\mathrm{a}, R, n}^{\dagger} a_{\mathrm{a}, R, n}+a_{\mathrm{a}, L, n}^{\dagger} a_{\mathrm{a}, L, n}\right)\right] .
$$

Imposing periodic boundary conditions on the lattice fermions leads to the quantization of the zero mode eigenvalues in the same way as in the $U=0$ case. In particular we again have

$$
e^{i \sqrt{4 \pi} Q_{\sigma}}=e^{-i \sqrt{4 \pi} \bar{Q}_{\sigma}}= \begin{cases}1 & \text { if } L=8 n+4 \\ -1 & \text { if } L=8 n\end{cases}
$$

The corresponding eigenvalues are the same as for $U=0$, see eqns 2.40, 2.50). 


\section{A. Finite-size spectrum for $L=8 n+4$}

Using the quantization conditions for the zero mode operators in the mode expansion 4.10 , we obtain a finite-size spectrum of the form

$$
\begin{aligned}
E & =\frac{2 \pi v_{c}}{\mathcal{L}}\left[\frac{1}{8 K}\left(m_{\uparrow}+m_{\downarrow}+m_{\uparrow}^{\prime}+m_{\downarrow}^{\prime}\right)^{2}+\frac{K}{8}\left(m_{\uparrow}+m_{\downarrow}-m_{\uparrow}^{\prime}-m_{\downarrow}^{\prime}\right)^{2}+\sum_{n>0} n\left[M_{n, c}^{+}+M_{n, c}^{-}\right]\right] \\
& +\frac{2 \pi v_{s}}{\mathcal{L}}\left[\left(\frac{m_{\uparrow}-m_{\downarrow}}{2}\right)^{2}+\left(\frac{m_{\uparrow}^{\prime}-m_{\downarrow}^{\prime}}{2}\right)^{2}+\sum_{n>0} n\left[M_{n, s}^{+}+M_{n, s}^{-}\right]\right] .
\end{aligned}
$$

Defining new quantum numbers

$$
\Delta N_{c}=m_{\uparrow}+m_{\downarrow}+m_{\uparrow}^{\prime}+m_{\downarrow}^{\prime}, \quad \Delta N_{s}=m_{\downarrow}+m_{\downarrow}^{\prime}, \quad 2 D_{c}=m_{\downarrow}-m_{\downarrow}^{\prime}, \quad 2 D_{s}=m_{\uparrow}-m_{\downarrow}-m_{\uparrow}^{\prime}+m_{\downarrow}^{\prime},
$$

and using that $K=\xi^{2} / 2$, we recover the expression 3.13 (with the correct selection rules for $D_{c, s}$ ) obtained directly from the Bethe Ansatz.

\section{B. Finite-size spectrum for $L=8 n$}

Here we can proceed analogously, the only difference being that we the eigenvalues are shifted

$$
m_{\sigma} \rightarrow m_{\sigma}-\frac{1}{2}, \quad m_{\sigma}^{\prime} \rightarrow m_{\sigma}^{\prime}-\frac{1}{2}
$$

The finite-size spectrum is then obtained by carrying out these substitions in Eqn 4.12 . Analysis of the resulting energy levels shows that there are four ground states with $E=\pi v_{s} / \mathcal{L}$ characterized by quantum numbers

$$
\begin{aligned}
& m_{\uparrow}=m_{\uparrow}^{\prime}=1, m_{\downarrow}=m_{\downarrow}^{\prime}=0, \\
& m_{\uparrow}=m_{\uparrow}^{\prime}=0, m_{\downarrow}=m_{\downarrow}^{\prime}=1, \\
& m_{\sigma}=m_{-\sigma}^{\prime}=1, m_{-\sigma}=m_{\sigma}^{\prime}=0 .
\end{aligned}
$$

All four states occur in the sector $N_{\uparrow}+N_{\downarrow}=L / 2$, but they differ in their $S^{z}$ eigenvalues. In particular, the last two states both have $S^{z}=0$.

\section{Ground State for $L=8 n$}

We know from our analysis of the Hubbard model, that the unique ground state occurs in the sector with $S^{z}=0$, and is part of a spin triplet. To order $\mathcal{O}\left(L^{-1}\right)$ this state is degenerate with a spin singlet, which explains the four-fold degeneracy observed in the Littinger liquid description. As we are ultimately interested in the ground state of the Hubbard model, we require an appropriate linear combination of the two states (4.15). Recalling that the zero mode eigenvalues are

$$
q_{\uparrow}=-q_{\downarrow}=\frac{\sqrt{\pi}}{2}, \quad \bar{q}_{\downarrow}=-\bar{q}_{\uparrow}=\frac{\sqrt{\pi}}{2} \quad \text { and } \quad q_{\uparrow}=-q_{\downarrow}=-\frac{\sqrt{\pi}}{2}, \quad \bar{q}_{\downarrow}=-\bar{q}_{\uparrow}=-\frac{\sqrt{\pi}}{2},
$$

we may use 2.60), 2.61) together with the requirement that the state must be symmetric under the interchange of up and down spins to conclude that the Hubbard model ground state for $L=8 n$ corresponds to

$$
|\mathrm{GS}\rangle \sim \cos \left(\sqrt{2 \pi} \Phi_{s}(0,0)\right)|0\rangle
$$

\section{Excited States for $L=8 n+4$}

For the tight-binding model we showed that correlators calculated in the particular excited states (2.23) for lattice lengths $L=8 n+4$ are essentially the same (apart from a shift in $k_{F}$ ) as ground state correlators for $L=8 n$. This 
suggests that for the Hubbard model there might be excited states on $L=8 n+4$ site lattices, whose EEs exhibit the same kind of additional contribution as the ground state of the $L=8 n$ site system. In order to investigate this idea, we consider the degenerate excited states characterized by the quantum numbers

$$
\begin{gathered}
\Delta N_{\uparrow}=\Delta N_{\downarrow}=1, \quad D_{\uparrow}=-D_{\downarrow}=\frac{1}{2}, \Leftrightarrow m_{\uparrow}=1, m_{\uparrow}^{\prime}=0, \quad m_{\downarrow}=0, m_{\downarrow}^{\prime}=1, \\
\Delta N_{\uparrow}=\Delta N_{\downarrow}=1, \quad D_{\uparrow}=-D_{\downarrow}=-\frac{1}{2}, \Leftrightarrow m_{\uparrow}=0, m_{\uparrow}^{\prime}=1, \quad m_{\downarrow}=1, m_{\downarrow}^{\prime}=0 .
\end{gathered}
$$

Both states are in the $S^{z}=0$ sector with two particles added relative to the ground state. The appropriate linear combinations that correspond to eigenstates of the total spin $\mathbf{S}^{2}$, i.e. spin triplet and singlet states, are

$$
\begin{aligned}
& \mid \text { triplet }\rangle \sim e^{-i \sqrt{2 \pi / K} \Theta_{c}(0,0)} \cos \left(\sqrt{2 \pi} \Phi_{s}(0,0)\right)|0\rangle, \\
& \mid \text { singlet }\rangle \sim e^{-i \sqrt{2 \pi / K} \Theta_{c}(0,0)} \sin \left(\sqrt{2 \pi} \Phi_{s}(0,0)\right)|0\rangle .
\end{aligned}
$$

In the following we will determine the entanglement entropies in these two states and compare them to their corresponding ground state values.

\section{E. Marginally irrelevant perturbation and " $L$-dependent exponents"}

In the bosonization approach reviewed above the Luttinger parameter in the spin sector is fixed by the $S U(2)$ symmetry to be $K_{s}=1$. It is however well known, that the spin sector is affected by the presence of a marginally irrelevant perturbation 12 , which gives rise to multiplicative logarithmic corrections in correlation functions of local operators 16 . A consequence of these corrections is that numerical results obtained for finite-size systems are generally not well described by the power-laws extracted from Luttinger liquid theory. This can be understood by employing renormalization group methods: the marginally irrelevant interaction essentially gives rise to a scale dependence of the spin Luttinger parameter. A proper treatment involves solving the appropriate Callan-Symanzik equation for the particular quantity of interest. This is beyond the scope of our work, and we instead resort to semi-phenomenological considerations. We take the lattice length $L$ to be our $\mathrm{RG}$ scale, and are interested in the regime $32 \leq L \leq 104$ relevant for our numerical studies of entanglement entropies. The idea is then to fit the finite-size ground state energy for $L=8 n$ to the form (see also Appendix A)

$$
\frac{E}{L}=\alpha+\beta L^{-2}
$$

For asymptotically large $L$ (so that the logarithmic corrections are negligible) the coefficient $\beta$ is related to the Luttinger parameter of the spin sector by

$$
K_{s}=\left(\pi v_{s}\right)^{-1}\left(\beta+\frac{\pi\left(v_{s}+v_{c}\right)}{6}\right)=1 .
$$

In presence of the marginal perturbation, the coefficient $\beta$ extracted from 4.20 becomes $L$ and $U$ dependent and we then can define an effective spin Luttinger parameter $K_{s}^{\text {eff }}(U)$ by

$$
K_{s}^{\mathrm{eff}}(U)=\left(\pi v_{s}\right)^{-1}\left(\beta+\frac{\pi\left(v_{s}+v_{c}\right)}{6}\right) .
$$

In the limit $L \rightarrow \infty$ we must have $K_{s}^{\text {eff }}(U)=1$ by $S U(2)$ symmetry. By analysing the ground state energy for a quarter-filled Hubbard chain for lattice lengths between $L=32$ and $L=104$ (which are the typical lengths considered in the numerical simulations in the following sections), we obtain the following results

$$
\begin{array}{l|l|l|l|l|l}
\hline \mathrm{U} & 0.5 & 1 & 2 & 4 & 16 \\
\hline K_{s}^{\text {eff }}(U) & 0.958 & 0.933 & 0.906 & 0.887 & 0.873 \\
\hline
\end{array}
$$

We note that, as expected, the effect of the marginally irrelevant corrections increases substantially with the Hubbard coupling $U$. 
The effects of the marginally irrelevant perturbation on the entanglement entropies for finite chains with $32 \leq L \leq$ 104 can then be estimated by replacing $K_{s}=1$ with $K_{s}^{\mathrm{eff}}(U)$. In particular, the calculation of the ground state entanglement would be modified by replacing the Luttinger liquid representation (4.17) of the ground state by

$$
\cos \left(\sqrt{2 \pi K_{s}^{\mathrm{eff}}(U)} \Phi_{s}(0,0)\right)|0\rangle
$$

Analogous replacement would be done for the excited states 4.19.

\section{CFT APPROACH TO ENTANGLEMENT ENTROPIES}

In the previous sections we have derived explicit representations of the ground state (and some low-lying excited states) of the quarter-filled Hubbard model in terms of the underlying bosonic CFT. The results are summarized as follows:

1. Ground state for $L=8 n+4$

$$
|\mathrm{GS}\rangle \sim|0\rangle .
$$

2. Ground state for $L=8 n$

$$
|\mathrm{GS}\rangle \sim \cos \left(\sqrt{2 \pi} \Phi_{s}(0,0)\right)|0\rangle \equiv \mathcal{O}_{1}|0\rangle .
$$

3. Excited states for $L=8 n+4$

$$
\begin{aligned}
\mid \text { triplet }\rangle \sim e^{-i \sqrt{2 \pi K} \Theta_{c}(0,0)} \cos \left(\sqrt{2 \pi} \Phi_{s}(0,0)\right)|0\rangle \equiv \mathcal{O}_{2}|0\rangle, \\
\mid \text { singlet }\rangle \sim e^{-i \sqrt{2 \pi K} \Theta_{c}(0,0)} \sin \left(\sqrt{2 \pi} \Phi_{s}(0,0)\right)|0\rangle \equiv \mathcal{O}_{3}|0\rangle
\end{aligned}
$$

The next step is to calculate entanglement properties within the CFT framework. In the first case (ground state for $L=8 n+4)$ the entanglement entropies follow from the general CFT vacuum result (1.4). In all other cases one is dealing with entanglement entropies of particular excited states (in the second case the state corresponds to an excitation on an extended Hilbert space, as discussed above). This observation allows us to make use of results for entanglement entropies in low-lying excited states in CFTs. A general approach to the the latter problem has been developed by Alcaraz et al ${ }^{17 / 18}$ (see also $\frac{19}{25}$ for other studies of the entanglement entropies in excited states of many body systems) and their main result can be summarized as follows. The n'th Rényi entropy for an excited state of the form $\mathcal{O}(0,0)|0\rangle$ is given by

$$
S_{n}=\frac{c}{6}\left(1+\frac{1}{n}\right) \ln \left[\frac{L}{\pi} \sin \left(\frac{\pi \ell}{L}\right)\right]+\frac{1}{1-n} \ln \left[F_{n}(\ell / L)\right]+c_{n}+o\left(L^{0}\right),
$$

where $c_{n}$ are $\mathcal{O}$-independent constants (which are the same as for the EEs in the CFT vacuum), and the scaling functions $F_{n}^{\mathcal{O}}(x)$ are given by

$$
F_{n}^{\mathcal{O}}(x)=\frac{\left\langle\prod_{k=0}^{n-1} \mathcal{O}\left(\frac{\pi}{n}(x+2 k)\right) \mathcal{O}^{\dagger}\left(\frac{\pi}{n}(-x+2 k)\right)\right\rangle}{n^{2 n(h+\bar{h})}\left\langle\mathcal{O}(\pi x) \mathcal{O}^{\dagger}(-\pi x)\right\rangle^{n}}
$$

Here $h$ and $\bar{h}$ are the conformal dimensions of the operator $\mathcal{O}$. For the Hubbard model, we have $c=2$ and are interested in the operators $\mathcal{O}_{j}(x)$ in Eqn $\sqrt{5.3}$. These operators factorize into a spin and a charge part, which in turn leads to the factorization of the scaling function $F_{n}^{\mathcal{O}}(x)$. Importantly, according to a result obtained by Alcaraz et al 17 the scaling function for vertex operators is trivial

$$
F_{n}^{e^{i \alpha \Theta_{c}}}(x)=1
$$

In our case this implies that the charge sector does not contribute to the scaling function. Furthermore, the structure of the expectation value of (5.5) is such that the results for $\mathcal{O}=\cos \left(\alpha \Phi_{s}\right)$ and $\mathcal{O}=\sin \left(\alpha \Phi_{s}\right)$ are identical. Combining these observations we conclude that the extra contribution in (5.4) is identical for the $L=8 n$ ground state (case 2) and the $L=8 n+4$ excitations (case 3) considered above. This provides a first prediction for Hubbard model EEs. 


\section{A. CFT calculation of the Rényi entropies}

In order to evaluate the correlation function appearing in the scaling function (5.5) we can use the standard Coulomb gas identity

$$
\left\langle\prod_{j=1}^{2 n}: 2 \cos \left(\beta \Phi_{s}\left(x_{j}\right)\right):\right\rangle=\sum_{\sigma_{1}, \ldots, \sigma_{2 n}} \delta_{\sigma_{1}+\sigma_{2}+\cdots+\sigma_{2 n}, 0} \prod_{i<j}\left|2 \sin \left(\frac{x_{i}-x_{j}}{2}\right)\right|^{\sigma_{i} \sigma_{j} \frac{\beta^{2}}{2 \pi}}
$$

where the $x_{j}$ 's can be read from Eq. (5.5) and they explicitly are

$$
x_{2 j-1}=\frac{\pi x}{n}+\frac{2 \pi(j-1)}{n}, \quad x_{2 j}=-\frac{\pi x}{n}+\frac{2 \pi(j-1)}{n}, \quad j=1, \ldots, n .
$$

Since in Eqs. 5.2 and 5.3 we have $\beta^{2}=2 \pi$, the function $F_{n}(x)$ can be readily obtained for low values of $n$ by direct computation, giving, up to $n=6$ :

$$
\begin{aligned}
& F_{2}(x)=\frac{1}{8}[7+\cos (2 \pi x)], \\
& F_{3}(x)=\frac{1}{9}[7+2 \cos (2 \pi x)], \\
& F_{4}(x)=\frac{1}{2048}[1435+604 \cos (2 \pi x)+9 \cos (4 \pi x)], \\
& F_{5}(x)=\frac{1}{625}[399+218 \cos (2 \pi x)+8 \cos (4 \pi x)], \\
& F_{6}(x)=\frac{1}{165888}[97430+64439 \cos (2 \pi x)+3994 \cos (4 \pi x)+25 \cos (6 \pi x)],
\end{aligned}
$$

where we introduced

$$
s_{n}=\sin \left(\frac{\pi x}{n}\right)
$$

It is worth mentioning that the expansion of $F_{n}(x)$ for small $x$ is

$$
F_{n}(x)=1+\frac{1}{6}\left(n-\frac{1}{n}\right)(\pi x)^{2}+O\left(x^{4}\right)
$$

in agreement with the general result reported in Ref 17 . We observe that our expressions for $F_{n}(x)$ for $1 \leq n \leq 6$ are equal to the square root of the scaling function $F_{n}^{\Upsilon}(x)$ for the operator $\Upsilon=i \partial \phi$ in a compactified boson theory, cf Eq. (56) in Ref. 18. Although we are unsure whether there is a deep connection between the two, we can safely conjecture that this relationship holds for arbitrary $n$, i.e.

$$
\left[F_{n}(x)\right]^{2}=F_{n}^{\Upsilon}(x)
$$

In Ref. 18 a determinant representation for the function $F_{n}^{\Upsilon}(x)$ has been obtained

$$
F_{n}^{\Upsilon}(x)=\left(\frac{1}{n} \sin (\pi x)\right)^{2 n} \operatorname{det} \mathbb{H} .
$$

where $\mathbb{H}$ is a $2 n \times 2 n$ matrix with elements

$$
\mathbb{H}_{j k}=\left\{\begin{array}{ll}
\frac{1}{\sin \left[\left(z_{j}-z_{k}\right) / 2\right]} & \text { if } j \neq k \\
0 & \text { if } j=k
\end{array} \text { and } \quad z_{j}= \begin{cases}\pi(2 j-2+x) / n & \text { if } j \leq n \\
\pi(2 j-2-x) / n & \text { if } j>n\end{cases}\right.
$$

This representation holds only for integer values of $n$, but in the next subsection we will provide its analytic continuation to arbitrary $n$. 


\section{B. The analytic continuation and the von Neumann Entropy}

In order to find the analytic continuation of $F_{n}(x)$ to arbitrary $n$, let us start by re-organising the order of row and column indices of the matrix $\mathbb{H}$ in Eq. (5.14) rewriting it in the block form

$$
\mathbb{H}=\left(\begin{array}{cc}
\mathbb{A} & \mathbb{B} \\
-\mathbb{B}^{T} & \mathbb{A}
\end{array}\right)
$$

where the matrix elements are $(i, j=1, \ldots, n)$

$$
\mathbb{A}_{i j}=\left\{\begin{array}{ll}
0 & \text { if } i=j, \\
\frac{1}{\sin [\pi(j-i) / n]} & \text { else },
\end{array}, \quad \mathbb{B}_{i j}=\frac{1}{\sin [\pi(j-i-x) / n]} .\right.
$$

It is straightforward to see that $\mathbb{A}$ and $\mathbb{B}$ commute and so

$$
\operatorname{det} \mathbb{H}=\operatorname{det}\left[\mathbb{A}^{2}+\mathbb{B}^{T} \mathbb{B}\right]
$$

A direct calculation shows also that

$$
\mathbb{B}^{T} \mathbb{B}=\alpha \mathbb{I},
$$

with $\mathbb{I}$ the $n \times n$ identity matrix and

$$
\alpha=\sum_{k=0}^{n-1} \frac{1}{\sin ^{2}[\pi(k+x) / n]}=\frac{n^{2}}{\sin ^{2} \pi x} .
$$

Furthermore the traces of the powers of $\mathbb{A}$ have also a particularly simple expression:

$$
\operatorname{Tr} \mathbb{A}^{2 k}=2(-)^{k} \sum_{p=1}^{\lfloor n / 2\rfloor}(n-2 p+1)^{2 k}
$$

It is then natural to expand det $\mathbb{H}$ in terms of these traces

$$
\begin{aligned}
\operatorname{det} \mathbb{H} & =\operatorname{det}\left(\alpha \mathbb{I}+\mathbb{A}^{2}\right)=\alpha^{n} \operatorname{det}\left(\mathbb{I}+\mathbb{A}^{2} / \alpha\right)=\alpha^{n} \exp \left[\operatorname{Tr} \sum_{k=1}^{\infty} \frac{(-)^{k+1}}{k} \frac{\mathbb{A}^{2 k}}{\alpha^{k}}=\alpha^{n} \exp \left[\sum_{k=1}^{\infty} \frac{(-)^{k+1}}{\alpha^{k} k} \operatorname{Tr} \mathbb{A}^{2 k}\right] .\right. \\
& =\alpha^{n} \exp \left[-\sum_{p=1}^{\lfloor n / 2\rfloor} 2 \sum_{k=1}^{\infty} \frac{1}{k}\left(\frac{(n-2 p+1)^{2}}{\alpha}\right)^{k}\right]=\alpha^{n} \prod_{p=1}^{\lfloor n / 2\rfloor} \exp \left[2 \ln \left(1-\frac{(n-2 p+1)^{2}}{\alpha}\right)\right] \\
& =\alpha^{n} \prod_{p=1}^{\lfloor n / 2\rfloor}\left(1-\frac{(n-2 p+1)^{2}}{\alpha}\right)^{2} .
\end{aligned}
$$

Finally, using the symmetry for $p \rightarrow n+1-p$ and the fact that for $n$ odd the term with $p=(n+1) / 2$ is 1 , we have

$$
\begin{aligned}
F_{n}^{\Upsilon}(x) & =\prod_{p=1}^{n}\left(1-\frac{(n-2 p+1)^{2}}{n^{2}} \sin ^{2}(\pi x)\right)=\left(\frac{2 \sin \pi x}{n}\right)^{2 n} \prod_{p=1}^{n}\left[\left(\frac{n}{2 \sin \pi x}\right)^{2}-\left(p-\frac{n+1}{2}\right)^{2}\right] \\
& =\left(\frac{2 \sin \pi x}{n}\right)^{2 n} \prod_{p=1}^{n}\left[\left(\frac{n}{2 \sin \pi x}\right)-\left(p-\frac{n+1}{2}\right)\right]\left[\left(\frac{n}{2 \sin \pi x}\right)+\left(p-\frac{n+1}{2}\right)\right] .
\end{aligned}
$$

Now we can use

$$
\prod_{p=1}^{n}(b+p)=\frac{\Gamma(1+b+n)}{\Gamma(1+b)}
$$


to write

$$
\begin{aligned}
F_{n}^{\Upsilon}(x) & =\left(\frac{2 \sin \pi x}{n}\right)^{2 n}(-1)^{n} \frac{\Gamma\left(1+\frac{1}{2}\left(\frac{n}{\sin \pi x}+n-1\right)\right) \Gamma\left(1-\frac{1}{2}\left(\frac{n}{\sin \pi x}-n+1\right)\right)}{\Gamma\left(1+\frac{1}{2}\left(\frac{n}{\sin \pi x}-n-1\right)\right) \Gamma\left(1-\frac{1}{2}\left(\frac{n}{\sin \pi x}+n+1\right)\right)} \\
& =\left(\frac{2 \sin (\pi x)}{n}\right)^{2 n} \frac{\Gamma^{2}\left(\frac{1+n+n \csc (\pi x)}{2}\right)}{\Gamma^{2}\left(\frac{1-n+n \csc (\pi x)}{2}\right)} .
\end{aligned}
$$

The result 5.24 allows us to deduce the following closed form expressions for the $\mathrm{n}^{\text {th }}$ Rényi entropies

$$
S_{n}=\frac{c}{6}\left(1+\frac{1}{n}\right) \ln \left[\frac{L}{\pi} \sin \left(\frac{\pi \ell}{L}\right)\right]+\frac{1}{1-n} \ln \left|\left(\frac{2 \sin (\pi \ell / L)}{n}\right)^{n} \frac{\Gamma\left(\frac{1+n+n \csc (\pi \ell / L)}{2}\right)}{\Gamma\left(\frac{1-n+n \csc (\pi \ell / L)}{2}\right)}\right|+c_{n}+o\left(L^{0}\right) .
$$

Furthermore, expanding (5.24) for small values of $x$ we explicitly recover Eq. (5.11). Finally, it is possible to take the derivative at $n=1$

$$
\left.\frac{\partial F_{n}^{\Upsilon}(x)}{\partial n}\right|_{n=1}=2\left[\ln |2 \sin (\pi x)|+\psi\left(\frac{1}{2 \sin (\pi x)}\right)+\sin (\pi x)\right]
$$

where we introduced $\psi(z)=\Gamma^{\prime}(z) / \Gamma(z)$ as the digamma function. Thus we conclude that the von Neumann entanglement entropy for the Hubbard model in the desired states is

$$
S_{1}=\frac{2}{3} \ln \left[\frac{L}{\pi} \sin \left(\frac{\pi \ell}{L}\right)\right]-g\left(\frac{\ell}{L}\right)+c_{1}+o(1)
$$

where

$$
g(x)=\log |2 \sin (\pi x)|+\psi\left(\frac{1}{2 \sin (\pi x)}\right)+\sin (\pi x)
$$

Third, the above analytic continuation also allows us to extract the limit for $n \rightarrow \infty$ of the Rényi entropy which corresponds to the logarithm of the maximum eigenvalue of the reduced density matrix, also known as single copy entanglement ${ }^{26}$. Taking explicitly the limit from Eq. 5.24 we obtain the scaling function

$$
f_{\infty}(x)=\lim _{n \rightarrow \infty}\left[\frac{1}{2(1-n)} \ln F_{n}^{\Upsilon}(x)\right]=1+\frac{(1-s) \ln (1-s)-(1+s) \ln (1+s)}{2 s},
$$

where $s=\sin (\pi x)$.

In Fig. 2 we report the CFT predictions for the scaling functions of the Rényi entropies as function of $x=\ell / L$. We note that while for small $x$ the scaling function is smaller for larger $n$, as given by the expansion (5.11), for $x=1 / 2$, i.e. in the middle of the chain, the behaviour is the opposite.

\section{Beyond CFT: effects of the marginal perturbation}

As we have already mentioned in Sec. IVE, the low-energy limit of the Hubbard model gives rise to a Luttinger liquid Hamiltonian (4.1), perturbed by a marginally irrelevant operator in the spin sector $\frac{12}{2}$. This leads to logarithmic corrections $^{16}$ in the finite size spectrum and correlation functions, which can be quite important when trying to compare analytic results to numerical computations on finite-size systems. The marginal perturbation is expected to induce logarithmic corrections to the entanglement entropies, and its effect on the ground state entanglement in CFTs was studied in some detail in Ref.27. The corrections to scaling for the ground state entanglement entropies turn out to be quite small for the Heisenberg chain 2933 , but they turn out to significantly affect the shell-filling effect in the Hubbard model as we will see below. A calculation of logarithmic corrections to the entanglement entropies of the quarter filled Hubbard model is significantly more difficult than for CFT ground states 27 , and certainly beyond the scope of our work. Instead, we will attempt to capture the effects of the marginally irrelevant perturbation phenomenologically as discussed in Sec. IVE by replacing the Luttinger parameter in the spin sector $K_{s}=1$ by an effective Luttinger parameter $K_{s}(L, U)$. This leads us to consider the entanglement in the modified state (4.24), 


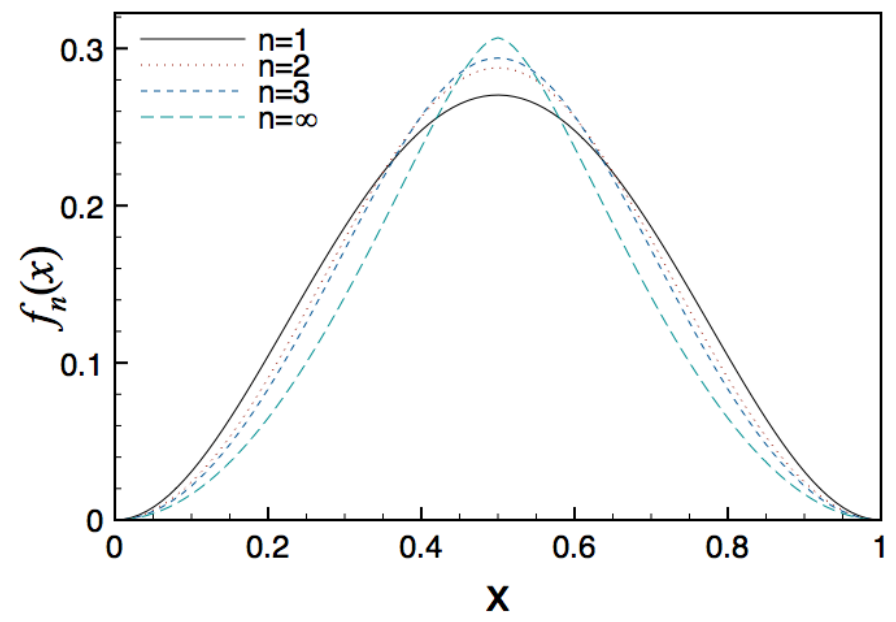

FIG. 2: The scaling function entering in the Rényi entropies $f_{n}(x)=\frac{1}{1-n} \ln F_{n}(x)$ for $n=1,2,3, \infty$ as function of $x=\ell / L$.

rather than the CFT state (5.2). The resulting phenomenological scaling function $F_{n}^{s}(x)$ can be calculated from the general expression 5.5 with $\mathcal{O}=\cos \left(\sqrt{2 \pi K_{s}^{\text {eff }}(U)} \Phi_{s}(0,0)\right)$. This resulting phenomenological scaling function $F_{2}^{s}(x)$ is of the form

$$
F_{2}^{s}(x)=\frac{1}{2}\left[1+\sin ^{4 K_{s}}\left(\frac{\pi x}{2}\right)+\cos ^{4 K_{s}}\left(\frac{\pi x}{2}\right)\right] .
$$

Analogous formulas for other small integer $n$ can be obtained, but the lack of a simple determinant representation for general values of $n$ precludes the determination of the corresponding von Neumann entropy.

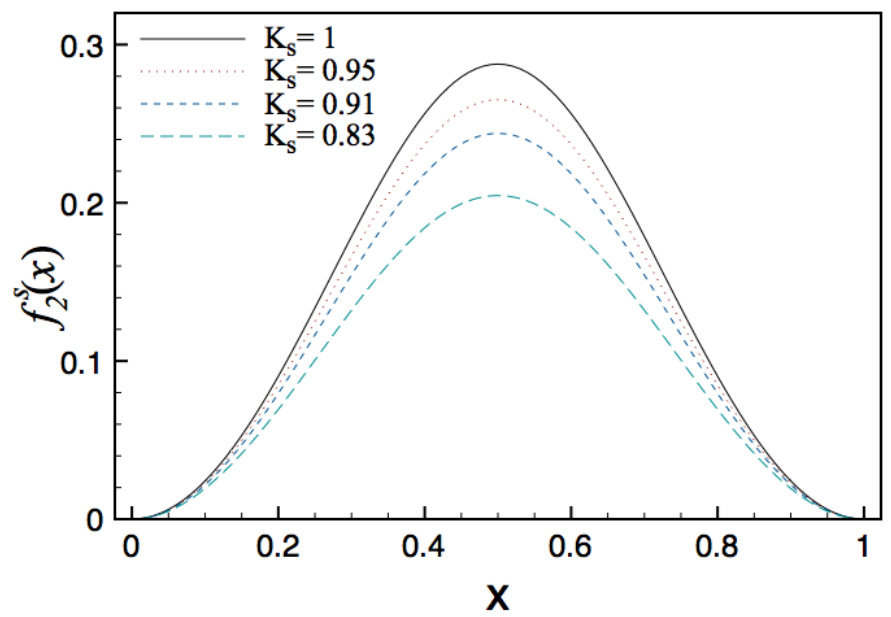

FIG. 3: The function $f_{2}^{s}(x)=-\ln F_{2}^{s}(x)$ for $K_{s}=1,0.95,0.91,0.83$ as function of $x=\ell / L$. We observe that a small variation of $K_{s}$ induces a significant change in $F_{2}^{s}(x)$.

In order to carry out comparisons to numerical results for EEs we use 5.30 and use the effective Luttinger parameter $K_{s}^{\mathrm{eff}}(U)$ determined in Sec. IV E from the finite size scaling of the ground state energy. In Fig. 3 we report the variation of $-\ln F_{2}^{s}(x)$ when $K_{s}$ varies form 1 to 1.2 which is the range of $K_{s}$ found in Eq. (4.23).

\section{COMPARISON TO NUMERICAL RESULTS}

We performed extensive DMRG ${ }^{28}$ computations of the periodic quarter-filled Hubbard model by keeping $M=3000$ states in order to achieve satisfactory convergence for periodic systems up to length $L=64$. In the following subsections we report the numerical results for several coupling parameters and lattice lengths running from $L=8$ 
to $L=64$, and covering both sequences of interest, i.e. $L=8 n$ and $L=8 n+4$. We perform detailed comparisons of these results with the CFT predictions obtained in the previous sections.

\section{A. Ground state EEs for $L=4 \bmod 8$.}

Let us start our analysis from $L=4 \bmod 8$, i.e. the lattice lengths which should give rise to a standard CFT result

$$
S_{n}^{\mathrm{CFT}}(\ell, L)=\frac{1}{3}\left[1+\frac{1}{n}\right] \ln \left[\frac{L}{\pi} \sin \left(\frac{\pi \ell}{L}\right)\right] .
$$

In this case the analysis is quite straightforward: in Fig. 4 we report the entanglement entropies $S_{1}$ (left panel) and $S_{2}$ (right panel) for $U=4 t$.
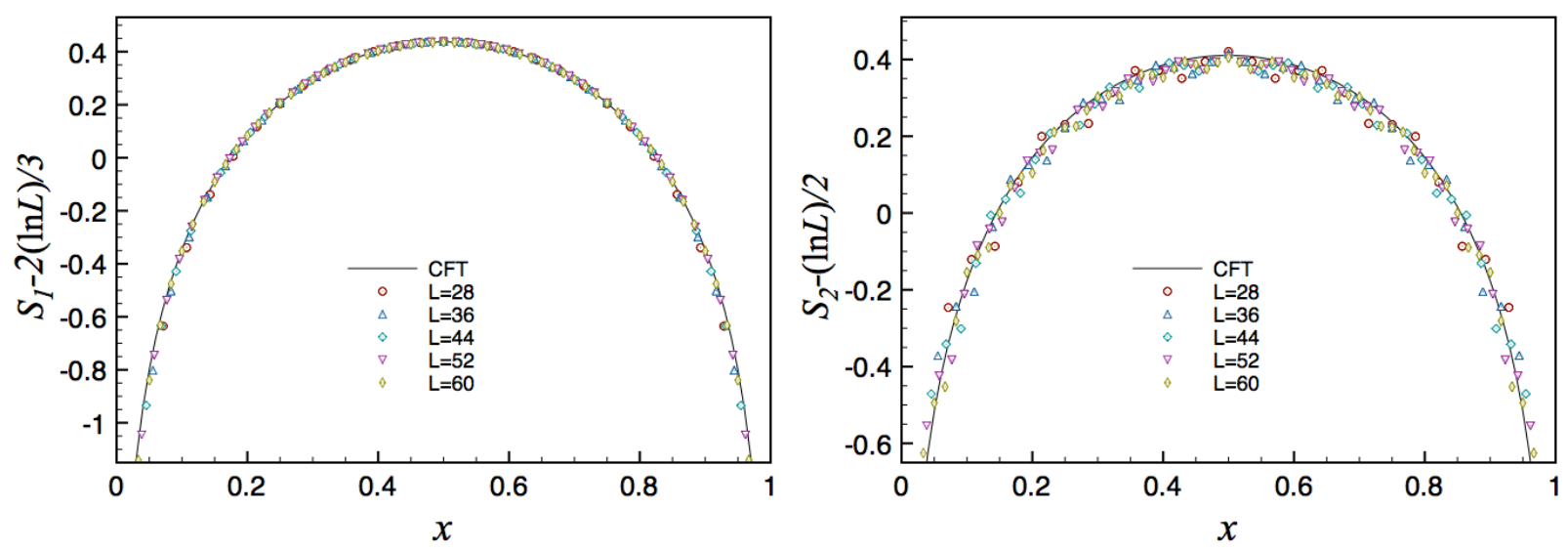

FIG. 4: Entanglement entropies as a function of $\ell / L$ for $U=4 t$ and $L=28,36,44,52,60$. We report $S_{n}-1 / 3(1+1 / n) \ln L$ for $n=1$ (left) and $n=2$ (right). The dots are the numerical results and the continuous lines the CFT prediction in Eq. 6.1) plus a non universal additive constant which is fixed by a fit. The agreement is excellent.

The data collapse and agreement between numerical data and the CFT prediction 6.1 are seen to be very good. In particular, for $n=1$ no significant deviations are apparent even for moderate lattice lengths. Conversely for $n=2$, the numerical data oscillate around the asymptotic result. This is as expected: the oscillations correspond to the well known "unusual corrections" $"[27 / 29 \sqrt{32}$ to the scaling of the entanglement entropies, and are known to be present for all Luttinger liquids and $n \neq 1$. We have verified that these corrections have the structure expected on the basis of the results obtained in Ref. ${ }^{29}$. Denoting by $S_{n}(\ell, L, U)$ the Rényi entropies of the Hubbard model at interaction strength $U$ on a periodic $L$-site chain, we consider the scaling of the quantity

$$
\delta S_{n} \equiv S_{n}(\ell, L, U)-S_{n}^{\mathrm{CFT}}(\ell, L),
$$

where $S_{n}^{\mathrm{CFT}}(\ell, L)$ is given in Eq. (145).

\section{B. Ground State EEs for $L=0 \bmod 8$.}

We now turn to lattice lengths $L=8 n$, for which we expect a (universal) $O(1)$ correction of the form (5.24) to the standard CFT result 6.1. In the following we first consider the von Neumann entropy and the turn to the second Rényi entropy.

\section{The von Neumann Entropy}

As we have already seen for the case $L=8 n+4$, the von Neumann entropy has the big advantage compared to the Rényi entropies that it does not contain pronounced oscillatory contributions. Its leading asymptotic behaviour for 
large $\ell$ and $L$ is given by the CFT result (5.27). According to the analysis of Sec. V] the corrections $\delta S_{1}(\mathrm{cf}(6.2))$ for large $L, \ell$ should converge to

$$
\delta S_{1}=-g(x)+c_{1}(U)
$$

where $g(x)$ is given in Eq. (5.28) and $c_{1}(U)$ is a non-universal $U$-dependent constant, which we use as a fitting parameter. The prediction (6.3) is compared to numerical results for $U=0.3 t, t, 4 t$ in Fig. 5 . For small values of $U$ $(U=0.3 t)$ the agreement is seen to be quite good, but there are increasingly large deviations when $U$ is increased.
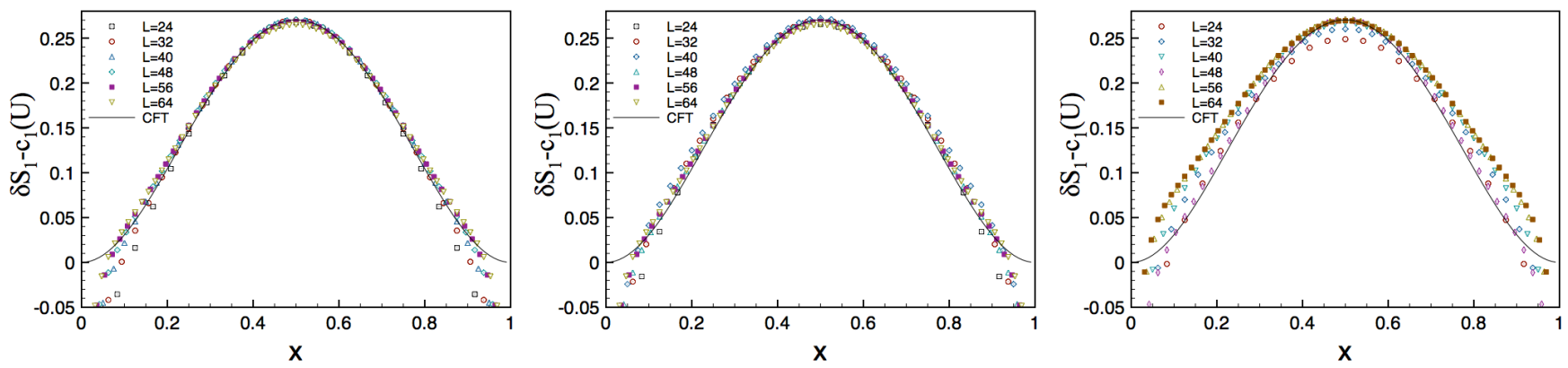

FIG. 5: Ground state EEs for $L=8 n$. We plot $\delta S_{1}-c_{1}(U)$ as a function of $x=\ell / L$ and $L=24,32,40,48,56,64$. The solid curve is the CFT prediction $-g(x)$. From left to right the three panels correspond to $U=0.3 t, U=t$, and $U=4 t$. The non universal constants are used as fitting parameters. Their values are $c_{1}(0.3)=1.205, c_{1}(1)=1.18, c_{1}(4)=1.14$.

An alternative analysis, which eliminates the unknown constants $c_{1}(U)$, is based on the fact that the latter are non-universal, but should not depend on the CFT state considered. In other words $c_{1}(U)$ is expected to be the same for EEs calculated in the ground state and in an excited state of the CFT 17 . In particular, $c_{1}(U)$ should be the same for $L=8 n$ and $L=8 n+4$. Hence, by subtracting the numerical data for $L=8 n$ from that for $L=8 n+4$, one should directly obtain the universal CFT function $g(\ell / L)$ (see also Refs ${ }^{17 / 18}$ ). Implementing such a procedure is not entirely straightforward, because the accessible values of $\ell / L$ differ for the two sequences of lattice lengths. We circumvent this problem as follows: since the data for the von Neumann entropy essentially lie on smooth curves (cf kFigs 5 ), we numerically interpolate the data for a given length in order to obtain a continuous function $\delta S_{1}^{\text {int }}(x, L)$ with $x \in(0,1)$. This allows us to compute the difference (here $L=8 n$ )

$$
\delta \tilde{S}_{1}(x, L) \equiv \delta S_{1}(\ell, L)-\delta S_{1}^{\mathrm{int}}(x, L-4) .
$$

Results for $\delta \tilde{S}_{1}(x, L)$ are shown in Fig. 6. We see that the deviations from the CFT prediction $-g(x)$ are still rather large for $U=t$ and $U=4 t$.
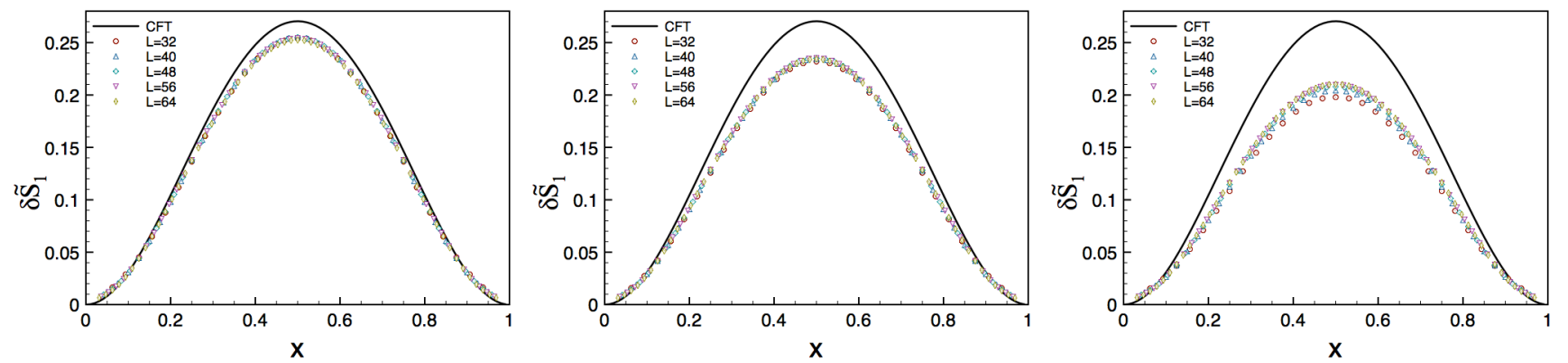

FIG. 6: $\delta \tilde{S}_{1}(x, L)$ for $L=32,40,48,56,64$ and as a function of $x=\ell / L$ for $U=0.3 t, t, 4 t$ from left to right. The solid line is the asymptotic CFT prediction.

\section{Origin of the observed deviations: cutoff effects.}

In this section we explore possible sources of the observed deviations between our numerical results and the CFT prediction (5.24) in particular for large values of the interaction strength $U$. 
The most basic issue to address is regime of applicability of the Luttinger liquid description to the Hubbard model. Field theory can be used to determine the behaviour of equal time correlation functions of local operators, as long as the separation between the latter are sufficiently large. The minimal requirement is that the separation needs to be large compared to the lattice spacing. For theories like the Hubbard model the situation is more complicated, because several degrees of freedom with different characteristic energy scales are involved. We can estimate the cutoff in the Luttinger liquid description as follows: we take as an energy cutoff the bandwidth $W_{s}$ of the spinon dispersion. At this energy scale deviations from a linear dispersion in the spin sector are clearly very large, and lattice effects dominate. We convert this energy scale to a length scale $\ell_{0}$ using the charge velocity, as the latter is always larger than the spin velocity for repulsive interactions, i.e.

$$
\ell_{0}=\frac{v_{c}}{W_{s}}
$$

The spinon and holon dispersions can be calculated exactly ${ }^{1}$, which leads to the following estimates for $\ell_{0}$ for a quarter-filled band

$$
\begin{array}{|l|l|l|l|l|}
U & v_{s} & v_{c} & W_{s} & \ell_{0} \\
\hline \hline 0.3 & 1.3667 & 1.4606 & 0.5835 & 2.50 \\
\hline 1 & 1.2589 & 1.5579 & 0.5655 & 2.75 \\
\hline 4 & 0.8818 & 1.8148 & 0.4297 & 4.22 \\
\hline 16 & 0.3444 & 1.9789 & 0.1721 & 11.5
\end{array}
$$

The Luttinger liquid description for equal time correlation functions of local operators is expected to be accurate at length scales large compared to $\ell_{0}$, i.e. $x \gg \ell_{0}$. As far as the finite-size entanglement entropy is concerned, the relevant length scale is the chord distance, and a rough estime for the applicability of the CFT results to the Hubbard model is then

$$
\frac{L}{\pi} \geq D(\ell, L) \gg \ell_{0}
$$

We see that for increasing $U$ the conformal description is expected to become worse and eventually ceases to apply for the available lattice lengths of $L \leq 60$. The upshot of these considerations is that for very large values of $U$ we should not expect good agreement between the CFT prediction and numerical results on lattices of $L \lesssim 60$ sites.

\section{Origin of the observed deviations: marginally irrelevant perturbation.}

A second source for the observed deviations is the presence of the marginally irrelevant perturbation in the spin sector. An immediate question that arises in such a scenario, is why this perturbation should strongly affect the $\mathcal{O}(1)$ contribtution for lattice lengths $L=8 n$, but appears to be negligible for $L=8 n+4$. First, we in fact expect the marginal perturbation to contribute to the $O(1)$ part of the ground state EEs for $L=8 n+4$, but as the latter is independent of the ratio $\ell / L$ it is quite difficult to spot this effect numerically. Second, it is known that marginal perturbations may have much more pronounced effects on corrections to excited state energies than to the ground state energy ${ }^{16}$. We conjecture that the corrections to the entanglement entropies behave in a similar way.

In order to investigate the possibility that the observed deviations between the numerical results and the CFT predictions (5.4), (5.9) are indeed caused by the marginally irrelevant perturbation in the spin sector, we now turn to the second Rényi entropy and implement the procedure set out in section VC. In analogy to the von Neumann entropy, we consider the scaling of the quantity $\delta S_{2}$ defined for general $n$ in Eq. (6.2).

The DMRG data for $\delta S_{2}(L, \ell)$ for coupling $U=0.3 t, t, 4 t, 16 t$ are reported in Fig. 7 for lattice lengths $L=$ $24,32,40,48,56,64$. In the various panels of Fig. 7 the dashed curves correspond to $c_{2}(U)-\ln \left(F_{2}(\ell / L)\right)$, where $c_{2}(U)$ is fixed by a fit. For $U=0.3 t$ the agreement is quite good and remaining discrepancies are compatible with arising from finite-size corrections, i.e. terms that vanish as $L \rightarrow \infty$ at fixed $\ell / L$. However, in analogy to what observed for the von Neumann entanglement entropy, the agreement becomes poorer increasing the values of $U$.

As discussed in section $\mathrm{VC}$, the effects of the marginal irrelevant perturbation on the second Rényi entropy can be taken into account by considering an effective Luttinger parameter $K_{s}$ in the spin sector $\underline{44}$. The resulting scaling function is (cf. Eq. (5.30)

$$
F_{2}^{s}(x)=\frac{1}{2}\left[1+\sin ^{4 K_{s}}\left(\frac{\pi x}{2}\right)+\cos ^{4 K_{s}}\left(\frac{\pi x}{2}\right)\right]
$$



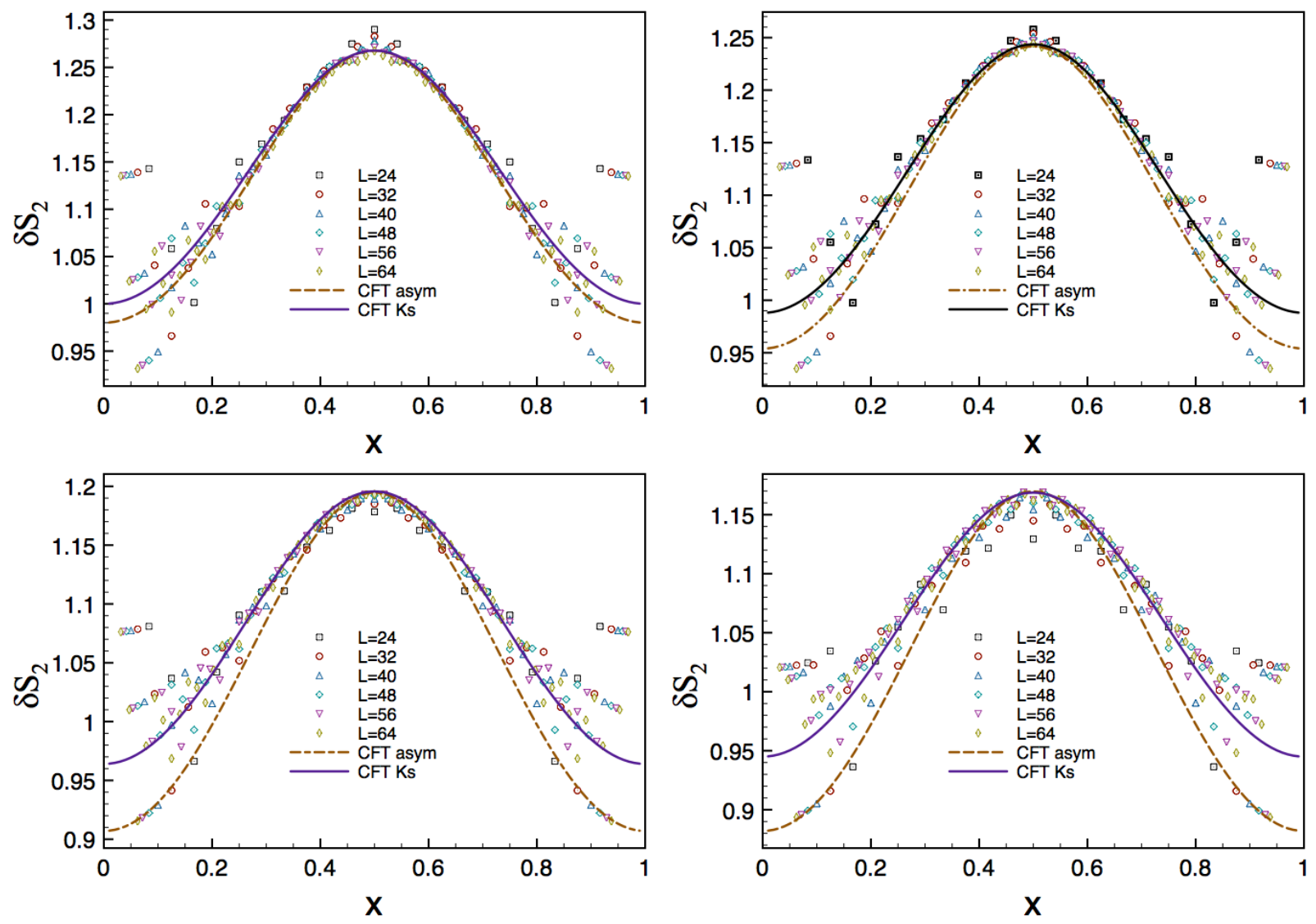

FIG. 7: Scaling of the second Rényi entropy. We plot $\delta S_{2}$ as a function of $x=\ell / L$ and for $L=24,32,40,48,56,64$. The different panels correspond to $U=0.3 t, t, 4 t, 16 t$. The dashed line is the function asymptotic CFT prediction $c_{2}(U)-\ln \left(F_{2}(\ell / L)\right)$. The continuous line is instead the "effective" CFT prediction in $c_{2}^{s}(U)-\ln \left(F_{2}^{s}(\ell / L)\right)$. The non-universal constant are used as fit parameters and are taken to be $c_{2}(0.3)=0.98, c_{2}(1)=0.954, c_{2}(4)=0.907$, and $c_{2}(16)=0.882$ in the CFT case, and $c_{2}^{s}(0.3)=1.00, c_{2}^{s}(1)=0.988, c_{2}^{s}(4)=0.964$, and $c_{2}^{s}(16)=0.945$ in "effective" CFT case.

In absence of renormalization group results we fix the effective Luttinger parameter $K_{s}^{\mathrm{eff}}(U, L)$ by the independent considerations discussed in section IVE, Eq. (4.23), in the range of lattice lengths $L$ relevant for our DMRG computations. More precisely, we compare $\delta S_{2}$ defined in Eq. 6.2 to

$$
c_{2}^{s}(U)-\ln F_{2}^{s}\left(\frac{\ell}{L}\right)
$$

where the constant $c_{2}^{s}(U)$ is the only fit parameter since $K_{s}$ is fixed in Eq. 4.23). The results of such an analysis are shown again in Fig. 7 as solid lines. We see that the agreement is now quite satisfactory for all values of $U$.

When the interaction strength $U$ is increased from 0 to $16 t$ for a $L \sim 60$ length lattice, the effective Luttinger parameter $K_{s}^{\text {eff }}(U, L)$ decreases from 1 to approximately 0.88 (cf. Eq. 4.23 ). This corresponds to a relatively moderate change of around 15\%. However, the function $F_{2}^{s}(x)$ is rather sensitive to this variation. Recalling the results of Fig. 3 we observe that in the middle of the chain $x=0.5$, the variation in $-\ln F_{2}^{s}(x)$ is approximately $40 \%$ when $K_{s}$ is decreased from $K_{s}=1$ to $K_{s}=0.88$. This sensitivity of the second Rényi entropy to changes in the Luttinger parameter is at the heart of the significant deviations between the CFT prediction and the numerics.

In our view, the analysis presented above constitutes important evidence in support of the idea that the main source of disagreement between the asymptotic CFT calculation and numerical data is the presence of a marginal irrelevant operator in the spin sector. 


\section{Excited State EEs for $L=4 \bmod 8$}

An key prediction of our theory for the shell-filling effect is that the additional contribution to the ground state EE for $L=8 n$ has the same functional form as the analogous contribution to a particular exited state EE for $L=8 n+4$. Here we test this prediction by comparing our CFT results to DMRG compuations. The relevant states for $L=8 n+4$ are the lowest excitations in the sector with two added particles and $S^{z}=0$ and could be either a spin triplet or a spin singlet, cf Eq. 5.3. According to the CFT approach (cf. Sec. V their EEs are the same and have the same functional form as the ground state EEs for $L=8 n$.

According to our CFT prediction, the difference

$$
\Delta S_{1}^{\mathrm{exc}}(\ell, L)=S_{1}^{\mathrm{exc}}(\ell, L)-S_{1}^{\mathrm{gs}}(\ell, L),
$$

for $L=8 n+4$ should converge to $-g(x)+c_{1}(U)$ for $L \rightarrow \infty$, where $g(x)$ is given in $[5.28)$. For small values of $U$ we find good agreement between this prediction and our numerical results.

On the other hand, for larger values of $U$ the situation mirrors that of the ground state EEs for $L=8 n$. Results for $\Delta S_{1}^{\text {exc }}$ for $U=4 t$ are shown in Fig. 8 (left). The agreement with the CFT prediction (solid line) at this value of $U$ is not particularly good, and in addition considerable finite-size effects are apparent. This is somewhat surprising,
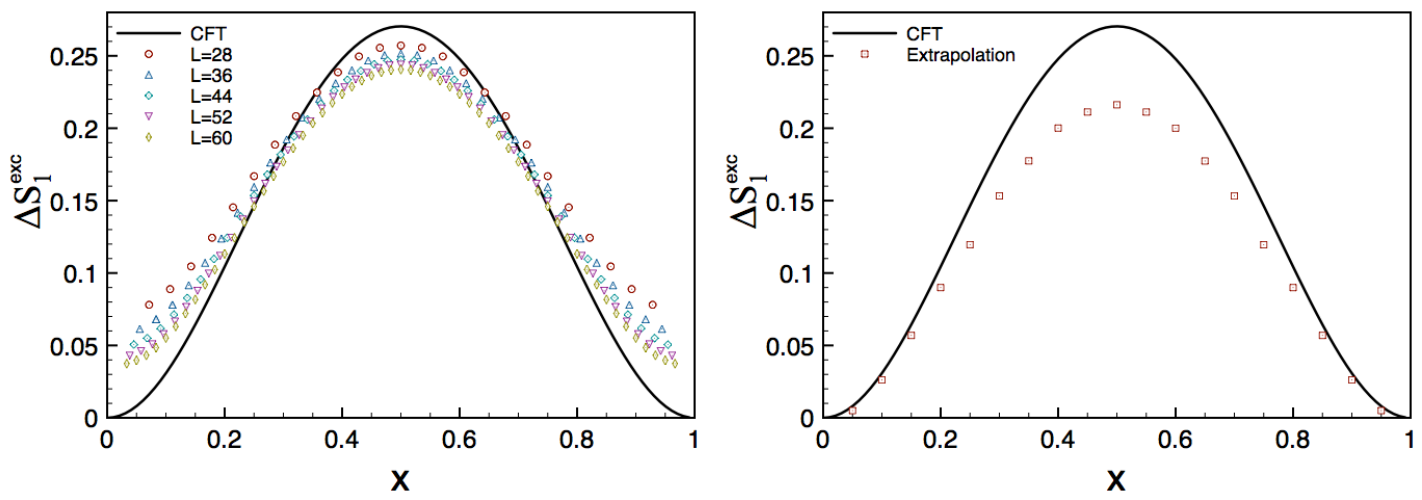

FIG. 8: Left: $\Delta S_{1}^{\operatorname{exc}}(\ell, L)$ as a function of $\ell / L$ for $U=4 t$ and $L=28,36,44,52,60$. Right: Extrapolation of $\Delta S_{1}^{\text {exc }}(\ell, L)$ in the left panel to large $L$. In both panels the solid line is the CFT prediction (5.27) for the difference.

as one might have expected a particularly good scaling collapse of the numerical data due to the fact that 6.10 involves EEs computed for the same lattice lengths. One reason for the pronounced finite-size effects is that ground and excited states differ by a shift of the Fermi momentum from $k_{F}=\pi / 4$ to $\tilde{k}_{F}=\pi / 4+\pi / L$ as a result of adding two particles. Plotting (6.10) at fixed $\ell / L$ thus gives rise to $\mathcal{O}\left(L^{-1}\right)$ corrections. A possible way to eliminate these, is to extrapolate the finite-size data. We do this as follows. We first interpolate the data for a given $L$ to obtain a continuous function $f_{L}$ of $x=\ell / L$. We then compute $f_{L}\left(x_{j}\right)$ for a selected set of points $x_{j} \in[0,1]$. Finally we carry out an extrapolation to infinite system size by fitting a second-order polynomial in $L^{-1}$ to the sequence $f_{L}\left(x_{j}\right)$ for a given $x_{j}$. The result of this procedure is shown in Fig. 8 (right). We see that there is a clear discrepancy between the extrapolated numerical and our theoretical prediction. This plot is quantitatively similar to the one for $U=4 t$ in Fig. 6 of the ground state EEs for $L=8 n$. This strongly suggests that the origin of the disagreement between the CFT prediction our numerical results is again the presence of a marginal irrelevant operator.

\section{ENTANGLEMENT ENTROPIES IN THE EXTENDED HUBBARD MODEL}

In the previous section we have accumulated evidence in favour of our claim, that the observed deviations between EEs in the Hubbard model and our CFT prediction (5.4) are caused by the presence of a marginally irrelevant perturbation in the spin sector. In order to remove any lingering doubts, we would like to numerically investigate a lattice model, for which the coupling constant of the marginally irrelevant perturbation can be tuned to zero (similar ideas have been employed in Ref $[35)$. This can be achieved by considering an extended quarter filled Hubbard model with Hamiltonian

$$
H_{\mathrm{ext}}=-t \sum_{j, \sigma}\left(c_{j, \sigma}^{\dagger} c_{j+1, \sigma}+c_{j+1, \sigma}^{\dagger} c_{j, \sigma}\right)-\mu \sum_{j} n_{j}+U \sum_{j} n_{j, \uparrow} n_{j, \downarrow}+\sum_{a=1}^{2} V_{a} \sum_{j} n_{j} n_{j+a} .
$$


This extended Hubbard chain has two additional coupling parameters $V_{1}$ and $V_{2}$ representing density-density interactions between nearest-neighbour and next-nearest neighbour sites. The model (7.1) is no longer integrable, but as long as $V_{1}$ and $V_{2}$ are not too large compared to $U,(7.1)$ is known to be in the same phase as the Hubbard chain (sec ${ }^{34}$ for the full phase diagram at small $V_{a}$ and quarter filling). Crucially, the interactions $V_{1,2}$ reduce the bare coupling constant $g_{1 \perp}$ of the marginally irrelevant interaction in the spin sector, at least at weak coupling. In fact, a perturbative calculation gives 34

$$
g_{1 \perp}=\frac{U a_{0}}{2}-V_{2} a_{0}-4 D_{1} a_{0}^{2}\left(\frac{U}{2}-V_{2}\right)\left(\frac{U}{2}-V_{1}+V_{2}\right),
$$

where $D_{1} \approx 1.25 /\left(8 \pi t a_{0}\right)$. Hence, at weak coupling, the most efficient way of reducing $g_{1 \perp}$ is to take

$$
V_{2} \sim U / 2
$$

We note that the nearest neighbour interaction cannot be used efficiently to this end, as the linear in $V_{1}$ contribution vanishes precisely for quarter filling as the above equation shows. At low energies and in the parameter regime of interest to us, the model (7.1) is described by a spin-charge separated Luttinger liquid, perturbed by a marginally irrelevant interaction in the spin sector, i.e.

$$
\mathcal{H}=\sum_{\alpha=c, s} \frac{v_{\alpha}}{2} \int d x\left[\left(\partial_{x} \Phi_{\alpha}\right)^{2}+\left(\partial_{x} \Theta_{\alpha}\right)^{2}\right]+\lambda\left(U, V_{2}\right) \int d x\left[\frac{2}{\pi a_{0}^{2}} \cos \left(\sqrt{8 \pi} \Phi_{s}\right)+\left(\partial_{x} \Theta_{s}\right)^{2}-\left(\partial_{x} \Phi_{s}\right)^{2}\right]
$$

Increasing $V_{2}$ from zero (and keeping $V_{1}=0$ throughout) leads to a Kosterlitz-Thouless transition at some critical value $V_{2, \text { crit }}$, which is characterized by

$$
\lambda\left(U, V_{2, \text { crit }}\right)=0
$$

So precisely at $V_{2 \text {,crit }}$ logarithmic corrections are absent. Moreover we expect logarithmic corrections to decrease when $V_{2}$ is increased from zero to $V_{2, \text { crit }}$.

DMRG results for the subtracted von Neumann entropy $\delta S_{1}$ (defined in Eq. (6.2) for $U=2 t$ and for $V_{2}=$ 0, 0.25t, 0.5t are shown in Fig. 9 and are compared with the asymptotic CFT formula (5.27). Clearly the evolution
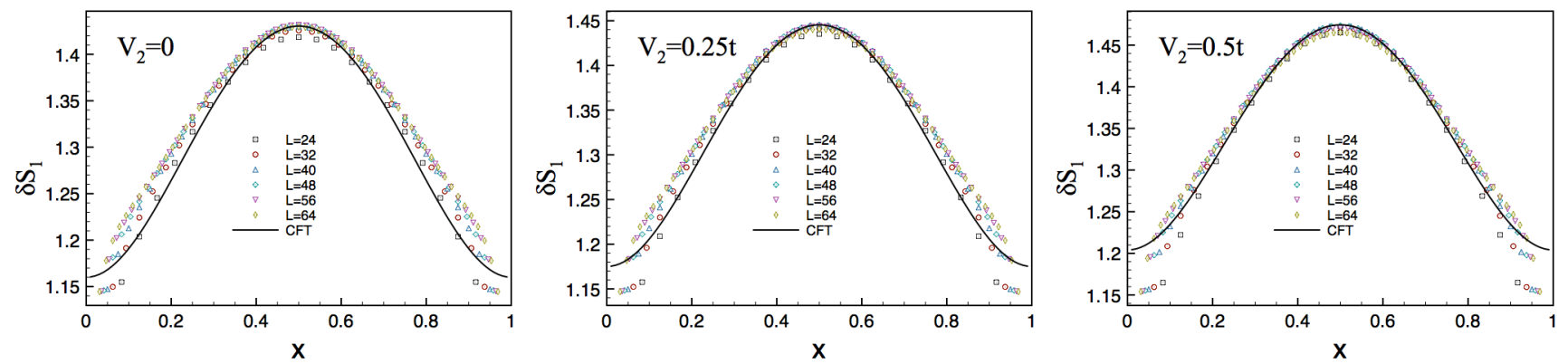

FIG. 9: $\delta S_{1}$ as a function of $\ell / L$ for $U=2 t$ and $V_{2}=0,0.25 t, 0.5 t$ from left to right. The solid line is the CFT prediction 5.27 .

of $\delta S_{1}$ with $V_{2}$ is in agreement with our expectation that the numerical results should approach the asymptotic CFT prediction as $V_{2}$ approaches $V_{2 \text {,crit }}$ from below.

\section{CONCLUSIONS}

We have presented a detailed analysis of the shell-filling effect in entanglement entropies of the quarter-filled one-dimensional Hubbard model with periodic boundary conditions. A short summary of our results has appeared previously in Ref.10]. The shell-filling effect, while somewhat unexpected, has a simple origin: for certain ratios of particle numbers to lattice length, the ground state in multi-component systems cannot be thought of in terms of a simple product of Fermi seas (in general these will consist of appropriate elementary excitations), but is in fact a linear combination of products of such seas.

By means of the Luttinger liquid representation of the Hubbard chain we developed a CFT approach to calculate the additional $\mathcal{O}(1)$ contribution to the Rényi entropies. These were found to be in very good agreement with results 
from DMRG computations for small values of the interaction strength $U$. For larger values of $U$ we found sizeable deviations between the CFT prediction and the DMRG data. We argued that these deviations can be explained by the presence of a marginally irrelevant perturbation in the spin sector. In simple cases such perturbations are known to give rise to logarithmic corrections to the entanglement entropies 27 . We substantiated this view by two complementary considerations. First, we demonstrated that taking the marginal perturbation into account semi-phenomenologically through a scale dependent effective Luttinger parameter in the spin sector leads to a good description of our numerical data for the second Rényi entropy. The analogous analysis for the von Neumann entropy is presently beyond reach, as the required analytic continuation in the Rényi index remains an open problem. Second, we verified that the shellfilling effect in an quarter-filled extended Hubbard chain, in which the coupling constant of the marginally irrelevant perturbation is reduced $\sqrt{34}$, is in better agreement with the CFT prediction.

We note that there are similarities between our results and those for EEs of linear combinations of degenerate ground states $36 \mid 37$. However, in our case the ground state is unique for $U>0$ (and fixed $S^{z}=0$ ) and the shell-filling effect does not a priori require a degeneracy.

The shell-filling effect is a rather general phenomenon as long as periodic boundary conditions are imposed. It is expected to be present also for other commensurate fillings in the Hubbard chain, multi-component continuum or lattice models of interacting fermions or Fermi-Bose mixtures, and higher dimensional critical systems. Examples of the former include multi-component gases with delta-function interactions $\sqrt{38}$ (which has been recently realized experimentally $\left.{ }^{40}\right)$, (extended) repulsive $S U(N)$ Hubbard or tJ models ${ }^{39}$. We believe that shell-filling effects may also come into play in numerical studies of two-dimensional gapless spin liquids, which display a spinon Fermi surface 41 .

\section{Acknowledgments}

We are grateful to F. Alcaraz and M. Fagotti for helpful discussions. This work was supported by the EPSRC under grants EP/I032487/1 and EP/J014885/1 (FHLE), the ERC under Starting Grant 279391 EDEQS (PC).

\section{Appendix A: Ground state energy for $L=8 n$ and logarithmic corrections}

In this Appendix we consider the effects of the marginally irrelevant interaction in the spin sector on the ground state energy. To that end, we numerically solve the Bethe Ansatz equations for lattices of up to $L=800$ sites for several values of $U$ and calculate the energy of the state $(3.19$. As a typical example for $U=4 t$ a fit to the ground state energy of the form

$$
\frac{E}{L}=\alpha+\beta L^{-2}
$$

where we consider the range $448 \leq L \leq L=768$, leads to coefficients

$$
\alpha=-0.758043, \quad \beta=1.16479 .
$$

If we only take into account lengths between $L=608$ and $L=768$ we obtain a slightly higher $\beta=1.16995$. The quality of the fit is quite good in either case: the fit residuals are of order $10^{-9}$ to $10^{-10}$. Now, if we use a fit function of the form

$$
\frac{E}{L}=\alpha+\frac{\beta}{L^{2}}+\frac{\gamma}{L^{2} \log (L / \delta)}
$$

we obtain an even better fit (residuals of order $10^{-12}$ ) with

$$
\alpha=-0.758044, \quad \beta=1.34988, \quad \gamma=1.16939, \quad \delta=1.56341 .
$$

In the thermodynamic limit we find by solving the integrals equations that

$$
e_{0}=-0.75804351, \quad-\pi \frac{v_{c}+v_{s}}{6}+\pi v_{s}=1.3584
$$

This suggests that it is crucial to take the logarithmic corrections into account: the agreement of $\beta$ with the thermodynamic value is poor if we neglect the logarithmic corrections, but it becomes quite good if we assume log corrections 
of the form $\mathrm{A} 3$. The strength of the logarithmic corrections depends on the value of $U$. Indeed, for $U=0.5 t$ a fit to A1 gives

$$
\alpha=-0.871464, \quad \beta=2.56928,
$$

while a fit of the same data to $\mathrm{A3}$ yields

$$
\alpha=-0.871464, \quad \beta=2.68008, \quad \gamma=1.14495, \quad \delta=87.5371 .
$$

The residuals are a factor of $10^{4}$ smaller in the logarithmic fit. From the solution of the integral equations describing the thermodynamic limit we have

$$
e_{0}=-0.87146392, \quad-\pi \frac{v_{c}+v_{s}}{6}+\pi v_{s}=2.71599 .
$$

The relative error in $\beta$ for the simple fit $\mathrm{A} 1$ is $5 \%$ for $U=0.5$, but $14 \%$ for $\mathrm{U}=4$.

${ }^{1}$ F. H. L. Essler, H. Frahm, F. Göhmann, A. Klümper, and V. E. Korepin, The One-Dimensional Hubbard Model, Cambridge University Press, (2005).

2 F. Woynarovich, J. Phys. A 22, 4243 (1989).

${ }^{3}$ L. Amico, R. Fazio, A. Osterloh, and V. Vedral, Rev. Mod. Phys, 80, 517 (2008);

J. Eisert, M. Cramer, and M. B. Plenio, ibid. 82, 277 (2010);

Entanglement entropy in extended systems, P. Calabrese, J. Cardy, and B. Doyon eds., J. Phys. A 42, 500301 (2009).

${ }^{4}$ V. E. Korepin, Phys. Rev. Lett. 92, 096402 (2004).

${ }^{5}$ C. Holzhey, F. Larsen, and F. Wilczek, Nucl. Phys. B 424, 443 (1994);

G. Vidal, J. I. Latorre, E. Rico, and A. Kitaev, Phys. Rev. Lett. 90, 227902 (2003);

J. I. Latorre, E. Rico, and G. Vidal, Quant. Inf. Comp. 4, 048 (2004).

${ }^{6}$ P. Calabrese and J. Cardy, J. Stat. Mech. P06002 (2004);

P. Calabrese and J. Cardy, J. Phys. A 42, 504005 (2009).

7 P. Calabrese and A. Lefevre, Phys. Rev. A 78, 032329 (2008).

8 L. Tagliacozzo, T. R. de Oliveira, S. Iblisdir, and J. I. Latorre, Phys. Rev. B 78, 024410 (2008);

F. Pollmann, S. Mukerjee, A. M. Turner, and J. E. Moore, Phys. Rev. Lett. 102, 255701 (2009).

9 J. I. Cirac and F. Verstraete, J. Phys. A 42, 504004 (2009);

G. Vidal, Entanglement Renormalization: an introduction, in Understanding Quantum Phase Transitions, ed. by L. D. Carr (Taylor \& Francis, Boca Raton, 2010) arXiv:0912.1651.

U. Schollwoeck, Ann. Phys. 326, 96 (2011)

10 F. H. L. Essler, A. M. Läuchli, and P. Calabrese, Phys. Rev. Lett. 110, 115701 (2013).

11 P. Broecker and S. Trebst, arXiv:1404.3027

12 I. Affleck, in Fields, Strings and Critical Phenomena, eds E. Brézin and J. Zinn-Justin, (Elsevier, Amsterdam, 1989); S. Eggert, in Theoretical Survey of One Dimensional Wire Systems, eds Y. Kuk et al., (Sowha Publishing, Seoul, 2007), arXiv:0708.0003

13 E. H. Lieb and F. Y. Wu, Phys. Rev. Lett. 20, 1445 (1968).

${ }^{14}$ F. H. L. Essler, V. E. Korepin and K. Schoutens, Phys. Rev. Lett. 67, 3848 (1991);

F. H. L. Essler, V. E. Korepin and K. Schoutens, Nucl. Phys. B 372, 559 (1992);

F. H. L. Essler, V. E. Korepin and K. Schoutens, Nucl. Phys. B 384, 431 (1992).

15 O. J. Heilmann and E. H. Lieb, Ann. N.Y. Acad. Sci. 172, 584 (1971);

C. N. Yang, Phys. Rev. Lett. 63, 2144 (1989).

16 J. L. Cardy, J. Phys. A 19, L109 (1986);

I. Affleck, D. Gepner, H. J. Schulz, and T. Ziman, J. Phys. A 22, 511 (1989);

I. Affleck, J. Phys. A 31, 4573 (1998);

S. Lukyanov, Nucl. Phys. B 522, 533 (1998).

17 F. C. Alcaraz, M. Ibanez Berganza, and G. Sierra, Phys. Rev. Lett. 106, 201601(2011).

18 F. C. Alcaraz, M. Ibanez Berganza, and G. Sierra, J. Stat. Mech. (2012) P01016.

19 V. Alba, M. Fagotti, and P. Calabrese, J. Stat. Mech. P10020 (2009).

${ }^{20}$ F. C. Alcaraz and M. S. Sarandy, Phys. Rev. A 78, 032319 (2008);

L. Masanes, Phys. Rev. A 80, 052104 (2009)

21 P. Calabrese, M. Mintchev, and E. Vicari, J. Stat. Mech. P09028 (2011);

L. Taddia, J. C. Xavier, F. C. Alcaraz, and G. Sierra, Phys. Rev. B 88, 075112 (2013).

${ }^{22}$ G.l Wong, I. Klich, L. A. Pando Zayas, and D. Vaman, JHEP 12 (2013) 020.

23 M. Nozaki, T. Numasawa, and T. Takayanagi, Phys. Rev. Lett. 112, 111602 (2014);

M. Nozaki, arXiv:1405.5875,

P. Caputa, M. Nozaki, and T. Takayanagi, arXiv:1405.5946 
${ }^{24}$ G. Ramirez, J. Rodriguez-Laguna, and G. Sierra, arXiv:1402.5015

Y. Huang and J. E. Moore, arXiv:1405.1817.

25 T. Palmai, arXiv: 1406.3182

26 J. Eisert and M. Cramer, Phys. Rev. A 72, 042112 (2005);

I. Peschel and J. Zhao, J. Stat. Mech. P11002 (2005).

27 J. Cardy and P. Calabrese, J. Stat. Mech. (2010) P04023.

28 S. R. White, Phys. Rev. Lett. 69, 2863 (1992);

U. Schollwöck, Rev. Mod. Phys. 77, 259 (2005).

29 P. Calabrese, M. Campostrini, F. Essler and B. Nienhuis, Phys. Rev. Lett. 104, 095701 (2010).

30 P. Calabrese and F.H.L. Essler, J. Stat. Mech. (2010) P08029.

31 N. Laflorencie, E. S. Sorensen, M.-S. Chang, and I. Affleck, Phys. Rev. Lett. 96, 100603 (2006);

M. Fagotti and P. Calabrese, J. Stat. Mech. P01017 (2011);

M. Dalmonte, E. Ercolessi, and L. Taddia, Phys. Rev. B 84, 085110 (2011);

J. C. Xavier and F. C. Alcaraz, Phys. Rev. B 85, 024418 (2012).

32 K. Ohmori and Y. Tachikawa, arXiv:1406.4167.

33 F. C. Alcaraz, unpublished.

${ }^{34}$ H. Yoshioka, M. Tsuchizu and Y. Suzumura, J. Phys. Soc. Jpn. 70, 762 (2001).

35 D. Schuricht, S. Andergassen and V. Meden, J. Phys. Cond. Mat. 25, 014003 (2013).

36 Y. Zhang, T. Grover, A. Turner, M. Oshikawa, and A. Vishwanath Phys. Rev. B 85, 235151 (2012).

37 O. A. Castro-Alvaredo and B. Doyon, Phys. Rev. Lett. 108, 120401 (2012);

V. Popkov, M. Salerno, and G. Schütz, Phys. Rev. E 72, 032327 (2005).

38 M. Gaudin, Phys. Lett. A24, 55 (1967);

C.N. Yang, Phys. Rev. Lett. 19, 1312 (1967);

M.A. Cazalilla and A. F. Ho, Phys. Rev. Lett. 91, 150493 (2003);

A. Imambekov and E. Demler, Phys. Rev. A73, 021602 (2006);

H. Frahm and G. Palacios, Phys. Rev. A72, 061604 (2005);

M.T. Batchelor, M. Bortz, X.W. Guan and N. Oelkers, Phys. Rev. A 72, 061603 (2005);

X.-W. Guan, M.T. Batchelor and C. Lee, arXiv:1301.6446.

39 P. Schlottmann, Int. J. Mod. Phys. B 11, 355 (1997).

40 G. Pagano, M. Mancini, G. Cappellini, P. Lombardi, F. Schafer, H. Hu, X.-J. Liu, J. Catani, C. Sias, M. Inguscio, L. Fallani, Nature Phys. 10, 198 (2014).

41 S.-S. Lee and P. A. Lee, Phys. Rev. Lett. 95, 036403 (2005).

42 O. L. Motrunich, Phys. Rev. B 72, 045105 (2005).

43 H.-Y. Yang, A.M. Läuchli, F. Mila, and K.P. Schmidt, Phys. Rev. Lett. 105, 267204 (2010).

44 As explained in section $\mathrm{VC}$ we are not currently able to implement a similar procedure for the von Neumann entropy. 